\title{
The spatial clustering of X-ray selected AGN in the XMM-COSMOS field
}

R. Gilli ${ }^{1}$, G. Zamorani ${ }^{1}$, T. Miyaji ${ }^{2}$, J. Silverman ${ }^{3}$, M. Brusa ${ }^{4}$, V. Mainieri ${ }^{5}$, N. Cappelluti ${ }^{4}$, E. Daddi ${ }^{6}$, C. Porciani $^{3}$, L. Pozzetti ${ }^{1}$, F. Civano ${ }^{7}$, A. Comastri ${ }^{1}$, A. Finoguenov ${ }^{4}$, F. Fiore ${ }^{8}$, M. Salvato ${ }^{9}$, C. Vignali ${ }^{10}$, G. Hasinger ${ }^{4}$, S. Lilly ${ }^{3}$, C. Impey ${ }^{11}$, J. Trump ${ }^{11}$, P. Capak ${ }^{9}$, H. McCracken ${ }^{12}$, N. Scoville ${ }^{9}$, Y. Taniguchi ${ }^{13}$, C. M. Carollo ${ }^{3}$, T. Contini ${ }^{14}$, J.-P. Kneib ${ }^{15}$, O. Le Fevre ${ }^{15}$, A. Renzini ${ }^{16}$, M. Scodeggio ${ }^{17}$, S. Bardelli ${ }^{1}$, M. Bolzonella ${ }^{1}$, A. Bongiorno ${ }^{4}$, K. Caputi ${ }^{3}$, A. Cimatti ${ }^{10}$, G. Coppa ${ }^{10}$, O. Cucciati ${ }^{18}$, S. de la Torre ${ }^{15}$, L. de Ravel ${ }^{15}$, P. Franzetti ${ }^{17}$, B. Garilli ${ }^{17}$, A. Iovino ${ }^{18}$, P. Kampczyk ${ }^{3}$, C. Knobel ${ }^{3}$, K. Kovač ${ }^{3}$, F. Lamareille ${ }^{14}$, J.-F. Le Borgne ${ }^{14}$, V. Le Brun ${ }^{15}$, C. Maier ${ }^{3}$, M. Mignoli ${ }^{1}$, R. Pellò ${ }^{14}$, Y. Peng ${ }^{3}$, E. Perez Montero ${ }^{14}$, E. Ricciardelli ${ }^{16}$, M. Tanaka ${ }^{5}$, L. Tasca ${ }^{15}$, L. Tresse ${ }^{15}$, D. Vergani ${ }^{1}$, E. Zucca ${ }^{1}$, U. Abbas ${ }^{15}$, D. Bottini ${ }^{17}$, A. Cappi ${ }^{1}$, P. Cassata ${ }^{15}$, M. Fumana ${ }^{17}$, L. Guzzo ${ }^{18}$, A. Leauthaud ${ }^{19}$, D. Maccagni ${ }^{17}$, C. Marinoni ${ }^{20}$, P. Memeo ${ }^{17}$, B. Meneux ${ }^{4}$, P. Oesch ${ }^{3}$, R. Scaramella ${ }^{8}$, and J. Walcher ${ }^{12}$

(Affiliations can be found after the references)

Received 18 August 2008 / Accepted 22 October 2008

\section{ABSTRACT}

We study the spatial clustering of $538 \mathrm{X}$-ray selected AGN in the $2 \mathrm{deg}^{2}$ XMM-COSMOS field that are spectroscopically identified with $I_{\mathrm{AB}}<23$ and span the redshift range $z=0.2-3.0$. The median redshift and X-ray luminosity of the sample are $z=0.98$ and $L_{0.5-10}=6.3 \times 10^{43} \mathrm{erg} \mathrm{s}^{-1}$, respectively. A strong clustering signal is detected at $\sim 18 \sigma$ level, which is the most significant measurement obtained to date for clustering of X-ray selected AGN. By fitting the projected correlation function $w\left(r_{\mathrm{p}}\right)$ with a power law on scales of $r_{\mathrm{p}}=0.3-40 h^{-1} \mathrm{Mpc}$, we derive a best-fit comoving correlation length of $r_{0}=8.6 \pm 0.5 h^{-1} \mathrm{Mpc}$ and slope of $\gamma=1.88 \pm 0.07$ (Poissonian errors; bootstrap errors are about a factor of 2 larger). An excess signal is observed in the range $r_{\mathrm{p}} \sim 5-15 h^{-1} \mathrm{Mpc}$, which is due to a large-scale structure at $z \sim 0.36$ containing about 40 AGN, a feature which is evident over many wavelengths in the COSMOS field. When removing the $z \sim 0.36$ structure or computing $w\left(r_{\mathrm{p}}\right)$ in a narrower range around the peak of the redshift distribution (e.g. $z=0.4-1.6$ ), the correlation length decreases to $r_{0} \sim 5-6 h^{-1}$ Mpc, which is consistent with what is observed for bright optical QSOs at the same redshift.

We investigate the clustering properties of obscured and unobscured AGN separately, adopting different definitions for the source obscuration. For the first time, we are able to provide a significant measurement for the spatial clustering of obscured AGN at $z \sim 1$. Within the statistical uncertainties, we do not find evidence that AGN with broad optical lines (BLAGN) cluster differently from AGN without broad optical lines (nonBLAGN). Based on these results, which are limited by object statistics, however, obscured and unobscured AGN are consistent with inhabiting similar environments.

The evolution of AGN clustering with redshift is also investigated. No significant difference is found between the clustering properties of XMM-COSMOS AGN at redshifts below or above $z=1$.

The correlation length measured for XMM-COSMOS AGN at $z \sim 1$ is similar to that of massive galaxies (stellar mass $M_{\star} \gtrsim 3 \times 10^{10} M_{\odot}$ ) at the same redshift. This suggests that AGN at $z \sim 1$ are preferentially hosted by massive galaxies, as observed both in the local and in the distant $(z \sim 2)$ Universe. According to a simple clustering evolution scenario, we find that the relics of AGN are expected to have a correlation length as large as $r_{0} \sim 8 h^{-1} \mathrm{Mpc}$ by $z=0$, and hence to be hosted by local bright $\left(L \sim L_{\star}\right)$ ellipticals.

We make use of dark matter halo catalogs from the Millennium simulation to determine the typical halo hosting moderately luminous $z \sim 1$ AGN. We find that XMM-COSMOS AGN live in halos with masses $M \gtrsim 2.5 \times 10^{12} M_{\odot} h^{-1}$. By combining the number density of XMM-COSMOS AGN to that of the hosting dark matter halos we estimate the AGN duty cycle and lifetimes. We find lifetimes approximately of 1 Gyr for AGN at $z \sim 1$, which are longer than those estimated for optically bright QSOs at the same redshift. These longer lifetimes mainly reflect the higher number density of AGN selected by X-ray samples.

Key words. galaxies: active - cosmology: large-scale structure of Universe - cosmology: observations - X-rays: galaxies

\section{Introduction}

Several pieces of evidence point towards an intimate correlation between the evolution of galaxies and the accretion and growth of supermassive black holes (SMBHs) at their centers, indicating that most galaxies in the Universe spent a fraction of their lifetimes as active galactic nuclei (AGN). In the local Universe, most galaxy bulges indeed host a supermassive black hole (see e.g. Ferrarese \& Ford 2005, for a review), whose mass scales with the bulge mass and stellar velocity dispersion (Ferrarese \& Merritt 2000; Gebhart et al. 2000). Furthermore, the growth of SMBHs during active accretion phases, which is traced by the cosmological evolution of the AGN luminosity function (Ueda et al. 2003; Hasinger et al. 2005; La Franca et al. 2005; Silverman et al. 2008a), has been shown to eventually match the mass function of SMBHs in the local Universe (e.g. Marconi et al. 2004; Yu \& Tremaine 2002; Shankar et al. 2004).

While the SMBH vs. galaxy co-evolution is now an accepted scenario, the details of this joint evolution are not fully understood yet. Nuclear activity in bright QSOs is thought to be induced by major mergers or close encounters of gas-rich 
galaxies in the context of hierarchical structure formation (e.g. Kauffmann \& Haehnelt 2000; Cavaliere \& Vittorini 2002; Hopkins et al. 2006). Alternatively, nuclear activity can be simply related to the physical processes (e.g. star formation) going on in a single galaxy, without being induced by mergers or interactions with neighboring objects (e.g. Granato et al. 2004). Overall, the role played by the environment in triggering both nuclear activity and star formation is still a matter of debate.

Just like local ultraluminous infrared galaxies (Sanders \& Mirabel 1996), the population of bright submillimeter sources at $z \sim 2$ (Chapman et al. 2003) is hosting both star formation and nuclear activity (Alexander et al. 2005), as a result of galaxy interactions (Tacconi et al. 2008). However, the majority of $z \sim 2$ AGN selected at faint X-ray fluxes seem to be hosted by galaxies with a spectral energy distribution typical of passively evolving objects (Mainieri et al. 2005). The concurrent growth of black holes and stellar mass has been observed in IR galaxies at $z \sim 2$ by Daddi et al. (2007), who suggested a long-lived (>0.2 Gyr) AGN plus starburst phenomenon, unlikely to be triggered by rapid merger events. In the local galaxies observed by the Sloan Digital Sky Survey (SDSS, York et al. 2000), nuclear activity does not appear to be correlated to the presence of close companions, while star formation does (Li et al. 2008). A common merger origin for both phenomena cannot be ruled out, however, provided they occur at different times (see Li et al. 2008).

The relation between nuclear activity and the environment can be studied via clustering techniques in the context of largescale structure formation, in which the growth of baryonic structures is supposed to follow the formations of dark matter halos (DMHs).

The comparison between the clustering properties of AGN and those of DMHs predicted by cold dark matter (CDM) models can be used to evaluate the typical mass of the DMHs in which AGN form and reside as a function of cosmic time. The most recent measurements have shown that bright QSOs in the redshift range $z=0-3$ reside into DMHs of mass $M>10^{12} M_{\odot}$ (Grazian et al. 2004; Porciani et al. 2004; Croom et al. 2005; but see Padmanabhan et al. 2008, for lower mass estimates at $z \sim 0.3)$. In addition, the ratio between the AGN space density and the space density of host DMHs may provide an estimate of the AGN lifetime (e.g. Martini \& Weinberg 2001). Current estimates are largely uncertain, constraining the AGN lifetime in the range of a few $\times 10^{6}-10^{8} \mathrm{yr}$ (Grazian et al. 2004; Porciani et al. 2004). Finally, the comparison between the clustering properties of different galaxy types and AGN can be used to estimate AGN hosts and to estimate the descendant and progenitors of AGN at any given redshifts.

AGN clustering has been traditionally studied by means of the two-point correlation function applied to optically selected QSO samples (e.g. Shanks et al. 1987; La Franca et al. 1998). The most recent and solid results of these analyses come from the two largest QSO surveys to date, namely the 2dF QSO Redshift Survey (2QZ, e.g. Croom et al. 2005), and the SDSS. The 2QZ is based on a sample of more than 20000 objects with redshifts $0.2 \lesssim z \lesssim 3.0$. When calculating the correlation function in real space and approximating it with a power law $\xi(r)=\left(r / r_{0}\right)^{-\gamma}$, the QSO correlation length and slope were found to be $r_{0}=5.0 \pm 0.5 h^{-1} \mathrm{Mpc}$ and $\gamma=1.85 \pm 0.13$ at a median redshift of $\bar{z}=1.5$ (Da Angela et al. 2005). Some evidence of a flattening towards smaller scales was also reported, with $\gamma=1.45$ at projected scales below $10 \mathrm{~h}^{-1} \mathrm{Mpc}$ (Da Angela et al. 2005). The clustering level of 2QZ QSOs is similar to that of early type galaxies at the same redshift (Coil et al. 2004; Meneux et al. 2006), suggesting they reside in environments of similar density.
A tentative detection of $z \sim 1$ AGN residing preferentially in the same environment of blue rather than red galaxies has been reported by Coil et al. (2007). The QSO clustering is observed to be a strong function of redshift (Croom et al. 2005; Porciani \& Norberg 2006), with the correlation length of luminous QSOs at $z \sim 4$ being as high as $r_{0}=24 h^{-1} \mathrm{Mpc}$ (Shen et al. 2007). This suggests that luminous, early QSOs are hosted by the most massive and rare DMHs and hence form in the highest density peaks of the dark matter distribution. The evidence of luminosity dependent clustering is, on the contrary, still marginal (Porciani \& Norberg 2006).

The above results are mostly based on AGN selected by means of their blue optical colors and broad optical lines; i.e., they essentially refer to unobscured, type 1 AGN. With the notable exception of the measurement performed by the SDSS on a local sample of narrow-line AGN (Li et al. 2006), to date there has been no information on the clustering properties of obscured AGN, which, based on the results from deep X-ray surveys (Brandt \& Hasinger 2005; Tozzi et al. 2006) and X-ray background synthesis models (e.g. Gilli et al. 2007a), are found to be a factor of $\gtrsim 4$ more numerous than unobscured ones; i.e., they are the most abundant AGN population in the Universe, dominating the history of accretion onto SMBHs (e.g. Fabian 1999). If the unified model strictly applies, i.e. the nuclear obscuration is just an orientation effect, one should not expect differences in the clustering properties of obscured and unobscured AGN. However, several exceptions to the strict unified model are known. Source obscuration is in many cases related to the gas content and evolutionary stage of the host galaxy, rather than to a small-scale torus intercepting the line of sight (Malkan et al. 1998). Models have been proposed in which the onset of nuclear activity starts embedded in an envelope of gas and dust, which is later on swept out by the QSO radiation (see e.g. Hopkins et al. 2006). If this were the case, obscured and unobscured AGN would be just two subsequent stages along a galaxy lifetime. The different durations of these two stages and their relation with the environment may produce different clustering properties between obscured and unobscured AGN.

One obvious way to obtain samples of obscured AGN is through X-ray observations. Besides reducing the obscuration bias dramatically, especially in the hard 2-10 keV band, X-ray selection also has the advantage of being effective in selecting distant low-luminosity AGN, whose optical light is diluted by the host galaxy emission and therefore missed by color-based optical surveys.

In the past years the limited sample size of X-ray selected AGN prevented clustering analyses as detailed as for optically selected objects. In particular, the lack of dedicated optical follow-up programs of X-ray sources providing large samples with spectroscopic measurements, has not allowed accurate estimates of the spatial clustering of X-ray selected AGN, limiting most studies to angular clustering. Numerous investigations of the two point angular correlation function of X-ray sources have indeed appeared in the literature, but the results suffer from rather large uncertainties. Early attempts to measure the angular clustering of X-ray selected sources were performed by Vikhlinin et al. (1995) and Carrera et al. (1998) based on ROSAT pointings. More recent results based on Chandra and XMM data have been obtained by Basilakos et al. (2004), Gandhi et al. (2006), Puccetti et al. (2006), Miyaji et al. (2007), Carrera et al. (2007), Plionis et al. (2008), and Ueda et al. (2008). In particular, Miyaji et al. (2007) and Gandhi et al. (2006) have computed the angular correlation function over contiguous areas of a few square degrees (the $2 \mathrm{deg}^{2}$ COSMOS field and the $\sim 4 \mathrm{deg}^{2}$ 
XMM-LSS field, respectively), which should reduce the impact of cosmic variance. In the COSMOS field, Miyaji et al. (2007) measured a correlation length of about $10 \pm 1 h^{-1} \mathrm{Mpc}$, while only a loose constraint $\left(r_{0}=3-9 h^{-1}\right) \mathrm{Mpc}$ was obtained in the XMM-LSS by Gandhi et al. (2006). Very recently an attempt to measure the angular clustering of high-redshift $(z \sim 3)$, X-ray selected AGN has been done by Francke et al. (2008).

The few examples of spatial clustering of X-ray selected sources appeared in the literature are limited by low statistics. Based on a sample of $\sim 220$ QSOs at $z \sim 0.2$ found in the $80 \mathrm{deg}^{2}$ ROSAT North Ecliptic Pole survey (NEP, Gioia et al. 2003), Mullis et al. (2004) were able to measure a correlation signal to $\gtrsim 3 \sigma$ level. By fixing the correlation slope to $\gamma=1.8$, they found a best-fit correlation length of $r_{0}=7.4_{-1.9}^{+1.8} h^{-1} \mathrm{Mpc}$. Because of the relatively short exposures in the NEP survey and the limited ROSAT sensitivity and bandpass $(0.1-2.4 \mathrm{keV})$, only bright, luminous, unobscured QSOs have been detected in

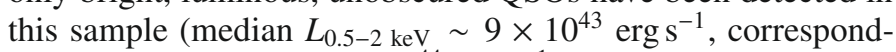
ing to $L_{0.5-10 \mathrm{keV}} \sim 2 \times 10^{44} \mathrm{erg} \mathrm{s}^{-1}$ for a spectral slope of $\alpha=0.9)$. Later, by analyzing data from the Chandra Deep Field South (CDFS, Rosati et al. 2002) and Chandra Deep Field North (CDFN, Alexander et al. 2003), Gilli et al. (2005) were able to detect clustering at $>7 \sigma$ level for $z \sim 0.7$ AGN with $L_{0.5-10 \mathrm{keV}} \sim 10^{43} \mathrm{erg} \mathrm{s}^{-1}$. However, the best-fit correlation length was found to vary by a factor of $\sim 2$ between the two fields $\left(r_{0}=10 h^{-1} \mathrm{Mpc}\right.$ in the CDFS, $r_{0}=5 h^{-1} \mathrm{Mpc}$ in the CDFN), revealing strong cosmic variance over these small, $0.1 \mathrm{deg}^{2}$ each, sky areas. Although with limited significance $(\sim 3 \sigma)$, in the CDFs, it was also possible to determine the clustering properties of obscured AGN only, which did not show significant differences with respect to those of unobscured ones within the uncertainties (Gilli et al. 2005). The most recent measurement is the one performed in the larger, $0.4 \mathrm{deg}^{2}$ field covered by the CLASXS (Yang et al. 2006). A correlation length of $\sim 5.7 h^{-1} \mathrm{Mpc}$ was found for X-ray selected AGN at $z \sim 1.2$, with average luminosity of $L_{0.5-10 \mathrm{keV}} \sim 6 \times 10^{43} \mathrm{erg} \mathrm{s}^{-1}$.

A large number of X-ray surveys are ongoing and are expected to provide larger samples of sources over wide sky areas and with different limiting fluxes. They will allow studies of clustering of AGN in different redshift and luminosity regimes. A few examples of such surveys are X-Bootes (Murray et al. 2005), XMM-LSS (Pierre et al. 2007), Extended CDFS (Lehmer et al. 2005), AEGIS (Nandra et al. 2005), and XMM-COSMOS (Hasinger et al. 2007). One of these samples, the XMM survey in the $2 \mathrm{deg}^{2}$ COSMOS field (XMM-COSMOS), has been specifically designed to study with the best statistics the clustering of X-ray selected AGN. One of the main goals of XMM-COSMOS was indeed to provide the best measurement to date of the correlation function of X-ray selected $\mathrm{AGN}$ and to allow a reliable measurement of the correlation function of obscured AGN at $z>0$ for the first time.

The optical spectroscopic identification of the 1822 pointlike X-ray sources detected by XMM-COSMOS continues. In this paper we present the results based on the first third of the objects. The paper is organized as follows. In Sect. 2 we summarize the X-ray and optical follow-up observations of the XMM-COSMOS sample and present the source catalog used in our analysis. In Sect. 3 we describe the methods of estimating the correlation function of X-ray selected sources. In Sect. 4 several safety checks are performed to validate the adopted techniques. The results of our analysis are presented in Sect. 5. In Sect. 6 the results are discussed and interpreted. The conclusions and prospects for future work are finally presented in Sect. 7.
Throughout this paper, a flat cosmology with $\Omega_{\mathrm{m}}=0.25$ and $\Omega_{\Lambda}=0.75$ is assumed (Spergel et al. 2007). For comparison with previous measurements we refer to correlation lengths and distances in units of $h^{-1} \mathrm{Mpc}$ comoving, where $H_{0}=$ $100 h \mathrm{~km} \mathrm{~s}^{-1} \mathrm{Mpc}^{-1}$. Masses of dark matter halos are also expressed in units of $h^{-1} M_{\odot}$ for consistency with the Millennium simulation, and AGN and halo space densities are expressed in units of $h^{3} \mathrm{Mpc}^{-3}$. AGN luminosities and lifetimes are calculated using $h=0.7$.

\section{The sample}

The XMM-COSMOS survey is part of the COSMOS legacy project (see Scoville et al. 2007a, for an overview of the survey), an extensive multiwavelength campaign to observe a $1.4 \times 1.4 \mathrm{deg}$ equatorial field centered at $(\mathrm{RA}, \mathrm{Dec})_{\mathrm{J} 2000}=$ $(150.1083,2.210)$. A number of large programs with the major observing facilities have been already performed or are ongoing, including HST (Scoville et al. 2007b), VLT (Lilly et al. 2007), SUBARU (Taniguchi et al. 2007), VLA (Schinnerer et al. 2007), XMM (Hasinger et al. 2007), Chandra (Elvis et al. 2009) Spitzer (Sanders et al. 2007), and GALEX (Zamojski et al. 2007; Schiminovich et al. in preparation).

XMM-COSMOS is a mosaic made of 53 partially overlapping XMM pointings that cover the entire $2 \mathrm{deg}^{2}$ COSMOS field. The XMM observations were allocated across two announcements of opportunities (AO-4 and AO-5) and performed in two different passes $^{1}$, for a total of $1.4 \mathrm{Ms}$ exposure time. Each pass was arranged into a regular grid of $\sim 30 \mathrm{ks}$ pointings separated by 8 arcmin each to cover the full $2 \mathrm{deg}^{2}$ field. In the second pass the grid pattern was shifted by 1 arcmin with respect to the first pass to ensure maximum uniformity in the sensitivity over the final mosaic. The limiting fluxes reached in the regions of maximum exposure are $5 \times 10^{-16}, 3 \times 10^{-15}, 6 \times 10^{-15} \mathrm{erg} \mathrm{cm}^{-2} \mathrm{~s}^{-1}$ in the $0.5-2,2-10$, and $5-10 \mathrm{keV}$, respectively, while the entire $2 \mathrm{deg}^{2}$ area is covered down to $2.4 \times 10^{-15}, 1.5 \times 10^{-14}$, $2 \times 10^{-14} \mathrm{erg} \mathrm{cm}^{-2} \mathrm{~s}^{-1}$ in the same bands. In total 1822 pointlike sources have been detected in at least one band down to a likelihood threshold of 10 (see Cappelluti et al. 2007, for the source detection process). The final catalog will be presented in a forthcoming paper (Cappelluti et al. 2009). A number of results concerning the first pass ( $0.8 \mathrm{Ms}$ total exposure) have been published by Cappelluti et al. (2007), Miyaji et al. (2007), and Mainieri et al. (2007).

The optical identification of XMM sources is currently in progress (see Brusa et al. 2007, for the initial results based on the first $12 \mathrm{XMM}$ pointings). Unique optical and/or infrared counterparts for most $(\sim 88 \%)$ of the XMM sources have now been recognized. Thanks to the Chandra observations in the central COSMOS square deg (Elvis et al. 2009), a number of formerly ambiguous identifications have now been made secure (Brusa et al. in preparation). The main dedicated spectroscopic follow-up programs of XMM sources are being conducted with the IMACS instrument at the $6 \mathrm{~m}$ Magellan telescope (Trump et al. 2007) and with VIMOS at the VLT within the zCOSMOS program (e.g. Lilly et al. 2007; see Fig. 1). A number of spectroscopic redshifts were also obtained by crosscorrelating the XMM catalog with published spectroscopic catalogs like the SDSS. About $46 \%$ of the total spectroscopic sample were obtained with only IMACS observations, $25 \%$ with only zCOSMOS, and another $24 \%$ has been observed in both

\footnotetext{
1 We do not consider here two additional pointings performed separately in AO-6.
} 


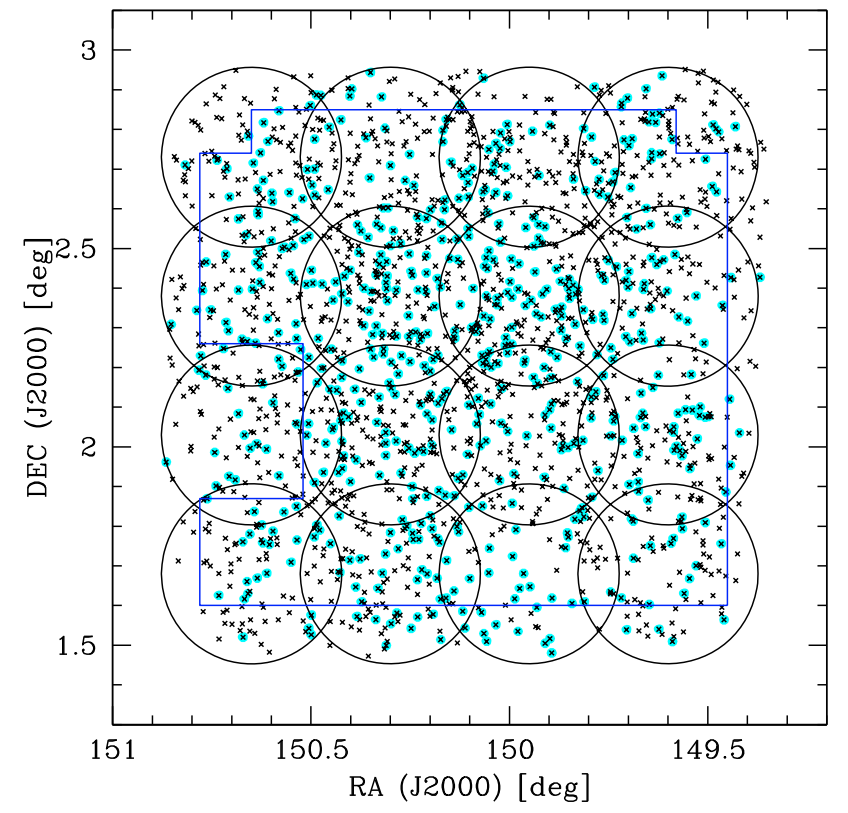

Fig. 1. Distribution on the sky of the 1822 pointlike sources detected by XMM (crosses). Spectroscopically identified objects are shown as filled circles. The area covered by spectroscopic observations is also shown: the large circles show the 16 Magellan IMACS pointings, while the polygon represents the area presently covered by zCOSMOS observations.

programs. The remaining $5 \%$ were obtained by cross-correlation with public catalogs. Quality flags were assigned to the redshifts measured by IMACS and zCOSMOS. We considered here only the $621 \mathrm{X}$-ray pointlike sources with highest quality flags, which have been identified as extragalactic objects. By considering the 150 duplicated redshifts (i.e. those objects observed by both IMACS and VIMOS) the accuracy in the redshift measurements is verified to be $\sigma_{z}<0.002$.

Since we combine measurements obtained with different optical instruments by different groups, which adopt somewhat different spectral classification criteria, it is not easy to obtain a uniform classification scheme for the optical counterparts of XMM sources. The only class with a common definition in the various catalogs is that of objects showing broad emission lines $\left(F W H M \gtrsim 2000 \mathrm{~km} \mathrm{~s}^{-1}\right)$. Therefore, in the following, as far as optical classification is concerned, we simply divide the sample into objects with and without broad optical lines, referring to them as broad line AGN (BLAGN) and non-BLAGN, respectively (see also Brusa et al. 2007).

The $0.5-2 \mathrm{keV}$ X-ray luminosity vs. redshift distribution of BLAGN and non-BLAGN, is shown in Fig. 2. BLAGN are on average observed at higher redshift and at luminosities above $10^{42.5} \mathrm{erg} \mathrm{s}^{-1}$, while non-BLAGN are observed down to very low X-ray luminosities and may therefore include a significant fraction of normal galaxies. To exclude from the sample those objects that are not likely to be AGN, we then considered only those sources at a redshift higher than 0.2. As shown in Fig. 2, this cut essentially removes most low-luminosity objects, leaving in the sample only objects with $L_{0.5-2} \gtrsim 10^{41.5} \mathrm{erg} \mathrm{s}^{-12}$. In addition we considered for our analysis only objects at redshifts below 3 , since beyond this limit the source density becomes extremely low and the selection function is very uncertain (see Sect. 3).

\footnotetext{
2 We verified that adopting a luminosity cut at $L_{0.5-10}>10^{41.5} \mathrm{erg} \mathrm{s}^{-1}$ produces similar results as adopting a redshift cut at $z=0.2$.
}

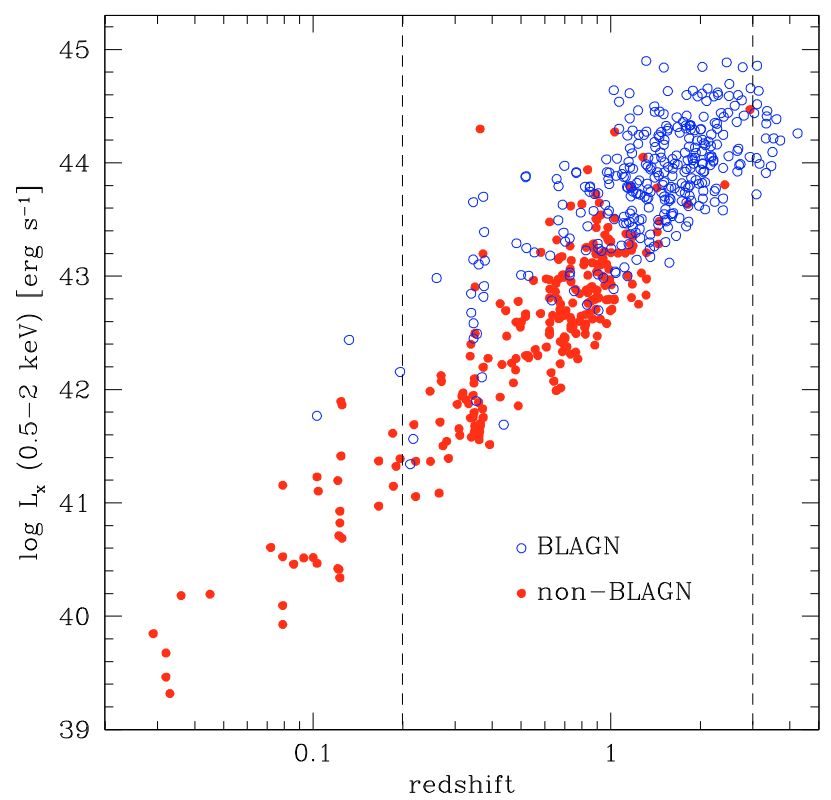

Fig. 2. Soft X-ray luminosity vs. redshift relation for the spectroscopically identified sources in XMM-COSMOS. Broad line AGN (BLAGN) and non broad line AGN (non-BLAGN) are shown as blue open circles and red filled circles, respectively. Only objects in the redshift range $z=0.2-3$ (vertical dashed lines) have been considered for the clustering analysis.

By exploiting the large multiwavelength database available in COSMOS, Salvato et al. (2009) are able to estimate a photometric redshift for $\sim 85 \%$ of the XMM sources. For the remaining $15 \%$ of the sample, the main reason for not attempting a photometric redshift estimate was the ambiguity in the correct association with the optical/IR counterpart (see Brusa et al. 2007). This issue, however, is not expected to introduce any bias related to source distances. Therefore the redshift distribution estimated using photometric redshifts should be very close to that of the entire XMM sample. On the contrary, spectroscopic redshifts have been measured for a minority $(\sim 36 \%)$ of the total XMM sample (including 34 stars), making it possible that objects with measured spectroscopic redshift are not a fair representation (i.e. a random sampling) of the total AGN population detected by XMM. The I-band magnitude vs. redshift distribution of the objects with spectroscopic redshifts and only photometric redshifts is shown in Fig. 3. Objects only with photometric redshift are on average optically fainter and at higher redshift than objects with spectroscopic redshifts. A Kolmogorov-Smirnov test performed on the redshifts distribution of objects with and without spectroscopic redshift indicates that they differ at $>3 \sigma$ level; i.e., the spectroscopic sample is not a fair representation of the entire AGN population detected by XMM. We therefore impose a magnitude cut at $I_{\mathrm{AB}}<23$, which excludes only a small fraction $(<3 \%)$ of spectroscopically identified objects but increases the spectroscopic completeness to about $60 \%$. The redshift distribution of objects brighter than $I_{\mathrm{AB}}=23$ with and without spectroscopic redshifts are statistically indistinguishable. Therefore, we conclude that, for objects with $I_{\mathrm{AB}}<23$, the spectroscopic selection does not include any bias against high-redshift objects.

In the following we consider the sample of $538 \mathrm{XMM}$ objects with $I_{\mathrm{AB}}<23$ and spectroscopic redshift in the range $z=0.2-3.0$ as our reference sample. The average redshift and 


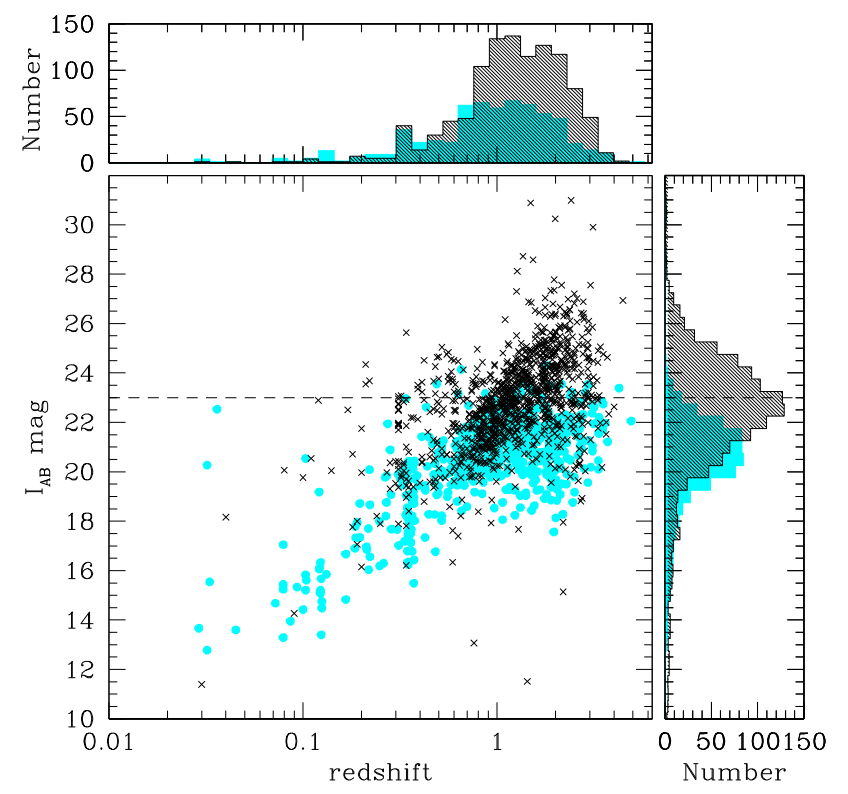

Fig. 3. I-band magnitude vs. redshift distribution of the optical counterparts of XMM sources. Objects with spectroscopic redshift or only photometric redshift are shown as filled circles or crosses, respectively. The dashed horizontal line shows the $I_{\mathrm{AB}}=23 \mathrm{mag}$ limit considered for the clustering analysis (see text).

X-ray luminosity of this sample are $z=0.98$ and $L_{0.5-10}=$ $6.3 \times 10^{43} \mathrm{erg} \mathrm{s}^{-1}$, respectively. The redshift distribution of the sample sources is shown in Fig. 4. A number of redshift structures are observed, the most prominent of which is at $z \sim 0.36$, also observed at other wavelengths in COSMOS (Lilly et al. 2007).

\section{Analysis techniques}

The basic statistics commonly used to measure the clustering properties of galaxies is the two point correlation function $\xi(r)$, defined as the excess probability over random of finding a pair with one object in the volume $\mathrm{d} V_{1}$ and the second in the volume $\mathrm{d} V_{2}$, separated by a distance $r$ (Peebles 1980):

$\mathrm{d} P=n^{2}[1+\xi(r)] \mathrm{d} V_{1} \mathrm{~d} V_{2}$,

where $n$ is the mean object space density. In our calculations we always refer to comoving distances and volumes. In a flat Universe one can simply estimate the comoving distance between two objects at redshifts $z_{1}$ and $z_{2}$ separated on the sky by an angle $\theta$ with the cosine rule (see e.g. Osmer et al. 1981):

$s=\sqrt{d_{1}^{2}+d_{2}^{2}-2 d_{1} d_{2} \cos \theta}$,

where $d_{i}$ is the radial comoving distance to object $i$, which, again in a flat universe, reads as

$d_{i}=\frac{c}{H_{0}} \int_{0}^{z_{i}} \mathrm{~d} z\left[\Omega_{\mathrm{m}}(1+z)^{3}+\Omega_{\Lambda}\right]^{-1 / 2}$.

However, a well known feature of Eq. (2) in measuring pair separations is that it is affected by redshift space distortions since peculiar velocities combine with the source distance to produce the measured redshift. (This is the same effect which in redshift surveys transforms galaxy clusters into fingers-of-God). The net effect of peculiar velocities is to shift pairs from smaller to larger radial separations, thus shifting the clustering power towards

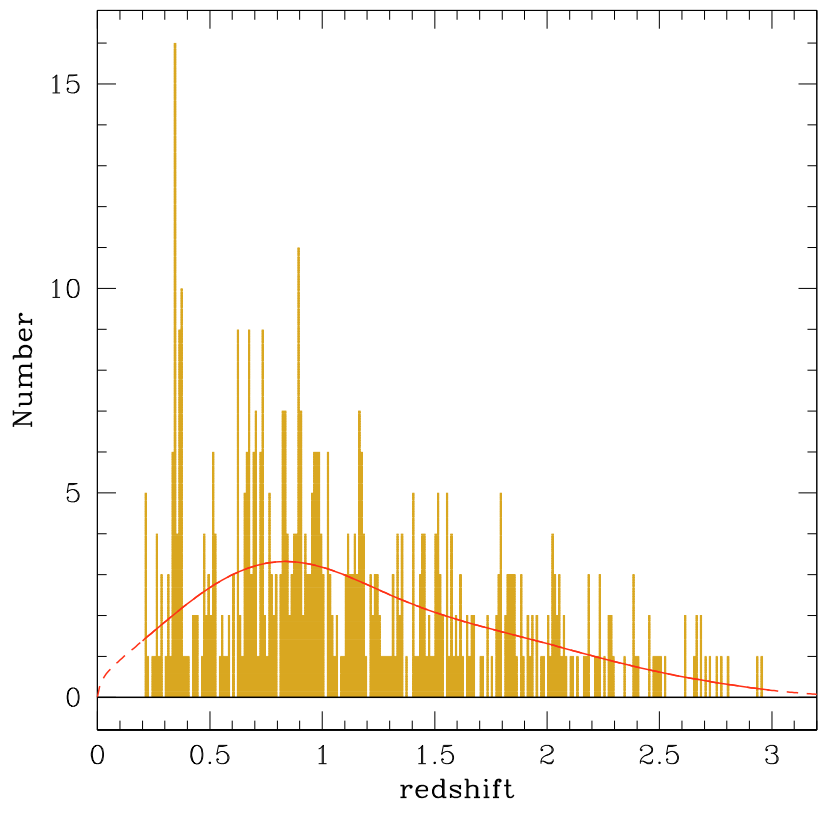

Fig. 4. Redshift distribution for the $538 \mathrm{X}$-ray sources in the reference sample in bins of $\Delta z=0.01$. The solid curve is obtained by smoothing the observed redshift distribution with a Gaussian with $\sigma_{z}=0.3$ and is used to generate the random control sample in the correlation function estimate.

higher scales. In particular a flatter slope with respect to the real space correlation function is generally observed. A similar effect is also produced by the uncertainties in the redshift measurements.

To overcome these problems one can resort to the so-called projected correlation function:

$w\left(r_{\mathrm{p}}\right)=\int_{-\pi_{\max }}^{\pi_{\max }} \xi\left(r_{\mathrm{p}}, \pi\right) \mathrm{d} \pi$,

where $\xi\left(r_{\mathrm{p}}, \pi\right)$ is the two-point correlation function expressed in terms of the separations perpendicular $\left(r_{\mathrm{p}}\right)$ and parallel $(\pi)$ to the line of sight as defined in Davis \& Peebles (1983) and applied to comoving coordinates:

$\pi=\left|d_{1}-d_{2}\right|, r_{\mathrm{p}}=\left(d_{1}+d_{2}\right) \tan \frac{\theta}{2}$.

Since $w\left(r_{\mathrm{p}}\right)$ is an integral along the radial coordinate, it is independent of peculiar velocity effects and can therefore be used as an estimate of the true, real-space correlation function. In particular, if the real space correlation function can be approximated by a power law of the form $\xi(r)=\left(r / r_{0}\right)^{-\gamma}$ and $\pi_{\max }=\infty$, then the following relation holds (Peebles 1980):

$w\left(r_{\mathrm{p}}\right)=A(\gamma) r_{0}^{\gamma} r_{\mathrm{p}}^{1-\gamma}$

where $A(\gamma)=\Gamma(1 / 2) \Gamma[(\gamma-1) / 2] / \Gamma(\gamma / 2)$ and $\Gamma(x)$ is the Euler's gamma function. Then, $A(\gamma)$ increases from 3.05 when $\gamma=2.0$ to 7.96 when $\gamma=1.3$.

An integration limit $\pi_{\max }$ has to be chosen in Eq. (4) to maximize the correlation signal. Indeed, one should avoid $\pi_{\max }$ values that are too high, since they would mainly add noise to the estimate of $w\left(r_{\mathrm{p}}\right)$. On the other hand, scales that are too small, comparable to the redshift uncertainties and to the pairwise velocity dispersion (i.e. the dispersion in the distribution of the relative velocities of source pairs), should also be avoided since they would not allow the whole signal to be recovered. 
The typical uncertainty in the redshift measurements $\left(\sigma_{z}<\right.$ 0.002 ) corresponds to comoving scales below $6.0 h^{-1} \mathrm{Mpc}$ at all redshifts. The pairwise velocity dispersion measured in the local Universe (500-600 $\mathrm{km} \mathrm{s}^{-1}$; Marzke et al. 1995; Zehavi et al. 2002) is expected to decrease by $\sim 15 \%$ at $z \sim 1.0$ (see e.g. the $\Lambda C D M$ simulations by Kauffmann et al. 1999), thus corresponding to $\sim 3 h^{-1} \mathrm{Mpc}$. To search for the best integration radius $\pi_{\max }$, we measured $w\left(r_{\mathrm{p}}\right)$ for the XMM-COSMOS reference sample for different $\pi_{\max }$ values ranging from 3 to $220 h^{-1}$ Mpc. In Fig. 5 (upper panel), we show the increase of $w\left(r_{\mathrm{p}}\right)$ with the integration radius $\pi_{\max }$ at those projected scales where most of the clustering signal is coming from $\left(r_{\mathrm{p}}=5-20 h^{-1} \mathrm{Mpc}\right)$. The $w\left(r_{\mathrm{p}}\right)$ values appear to converge for $\pi_{\max } \gtrsim 40 h^{-1} \mathrm{Mpc}$. Similarly, the amplitude of the spatial correlation function $B=r_{0}^{\gamma}$ (Fig. 5 bottom panel) or the amplitude of the projected correlation function $C=A(\gamma) r_{0}^{\gamma}$ (not shown) is converging for $\pi_{\max } \gtrsim 40 h^{-1} \mathrm{Mpc}^{3}$. The correlation length $r_{0}$ and slope $\gamma$ are strongly correlated: when $r_{0}$ increases, $\gamma$ decreases. The correlation length appears to reach a maximum at $\pi_{\max } \sim 80 h^{-1} \mathrm{Mpc}$, while $\gamma$ is constant in the range $\pi_{\max }=40-200 h^{-1} \mathrm{Mpc}$. Based on these considerations, we adopt $\pi_{\max }=40 h^{-1} \mathrm{Mpc}$ in the following analysis, which is the minimum $\pi_{\max }$ value at which the correlation function converges, and returns the smaller errors on the bestfit correlation parameters $r_{0}, \gamma$ and $r_{0}^{\gamma}$. We note that with this choice $r_{0}$ is smaller by $\sim 10 \%$ than the maximum value measured at $\pi_{\max } \sim 80 h^{-1} \mathrm{Mpc}$, but we do not try to correct for this small bias.

To measure $\xi\left(r_{\mathrm{p}}, \pi\right)$ we created random samples of sources in our fields and measured the excess of pairs at separations $\left(r_{\mathrm{p}}, \pi\right)$ with respect to the random distribution. We used the minimum variance estimator proposed by Landy \& Szalay (1993), which is found to have a nearly Poissonian variance and to outperform other popular estimators, especially on large scales (e.g., see Kerscher et al. 2000):

$\xi\left(r_{\mathrm{p}}, \pi\right)=\frac{[D D]-2[D R]+[R R]}{[R R]}$,

where $[D D],[D R]$ and $[R R]$ are the normalized data-data, datarandom and random-random pairs, i.e.

$[D D] \equiv D D\left(r_{\mathrm{p}}, \pi\right) \frac{n_{r}\left(n_{r}-1\right)}{n_{d}\left(n_{d}-1\right)}$

$[D R] \equiv D R\left(r_{\mathrm{p}}, \pi\right) \frac{\left(n_{r}-1\right)}{2 n_{d}}$

$[R R] \equiv R R\left(r_{\mathrm{p}}, \pi\right)$

where $D D, D R$, and $R R$ are the number of data-data, datarandom, and random-random pairs at separations $r_{\mathrm{p}} \pm \Delta r_{\mathrm{p}}$ and $\pi \pm \Delta \pi$, and $n_{d}$ and $n_{r}$ are the total number of sources in the data and random sample, respectively.

Since both the redshift and the coordinate $(\alpha, \delta)$ distributions of the identified sources are potentially affected by observational biases, special care has to be taken in creating the sample of random sources. This has been extensively discussed by Gilli et al. (2005) for the Chandra Msec Fields (see. e.g. their Sect. 4.2) where similar problems have been encountered. In that paper we showed that extracting the coordinates of the random sources from the coordinate ensemble of the real sample and

\footnotetext{
3 Since $A(\gamma)$ varies only by $\sim 16 \%$ in the range of the measured slopes,
} $B$ and $C$ show almost exactly the same behaviour with $\pi_{\max }$.

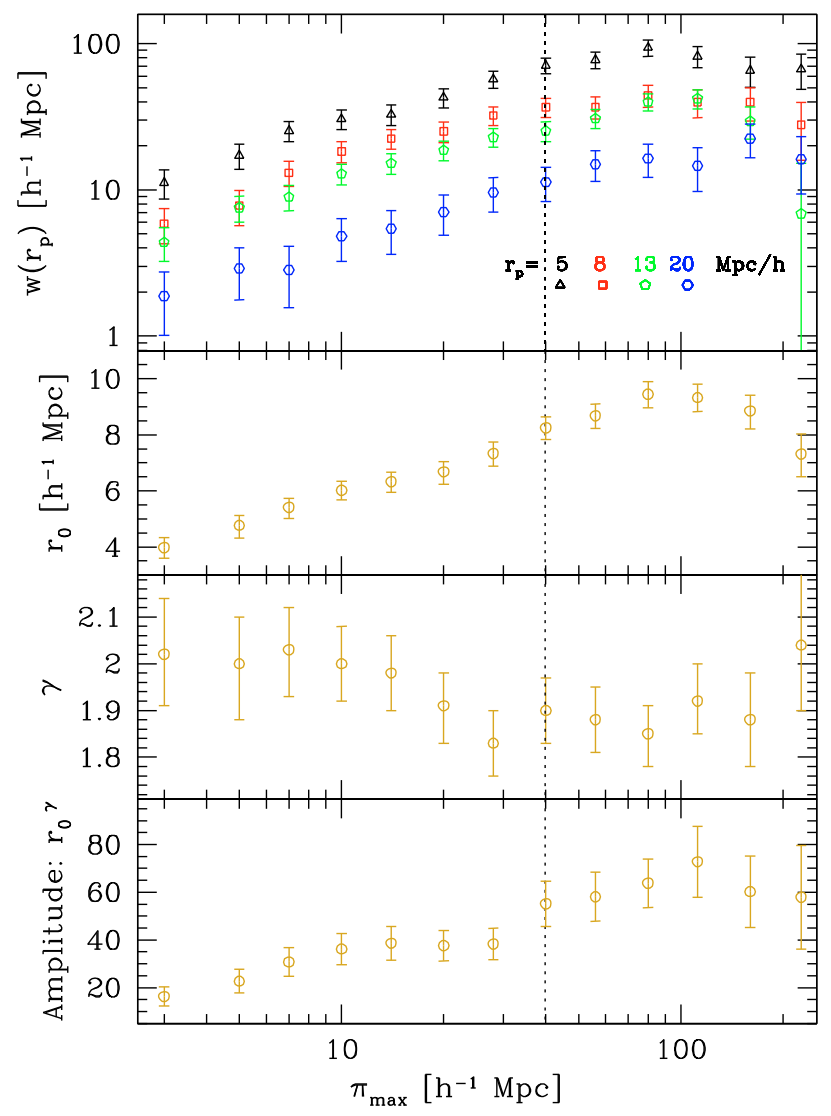

Fig. 5. Upper panel: projected correlation function $w\left(r_{\mathrm{p}}\right)$ computed at different $r_{\mathrm{p}}$ scales (see label) as a function of the integration radius $\pi_{\max }$. From top middle to bottom panels the correlation length $r_{0}$, slope $\gamma$, and amplitude $r_{0}^{\gamma}$ are shown as a function of $\pi_{\max }$, respectively.

using the observed redshifts to create a smoothed redshift distribution for the random sample is a sufficiently accurate procedure. As in Gilli et al. (2005), we assumed a Gaussian smoothing length $\sigma_{z}=0.3$, which is a good compromise between scales that are too small, which would suffer from local density variations, and those that are too large, which would oversmooth the distribution. We nonetheless verified that our results do not change significantly when using a smoothing length in the range $\sigma_{z}=0.2-0.4$. The smoothed redshift distribution adopted for our simulations is shown in Fig. 4. The adopted procedure, if anything, would slightly reduce the correlation signal, since it removes the effects of angular clustering. Each random sample is built to contain more than 20000 objects.

We binned the source pairs in intervals of $\Delta \log r_{\mathrm{p}}=0.2$ and measured $w\left(r_{\mathrm{p}}\right)$ in each bin. The resulting datapoints were then fitted by a power law of the form given in Eq. (6), and the bestfit parameters $\gamma$ and $r_{0}$ were determined via $\chi^{2}$ minimization. Given the small number of pairs that fall into some bins (especially on the smallest scales), we used the formulae of Gehrels (1986) to estimate the $68 \%$ confidence interval (i.e. $1 \sigma$ errorbars in Gaussian statistics).

\section{Safety checks and error estimates}

A possible concern related to the analysis methods presented in the previous section is the random-sample generation. Indeed, placing the random sources at the coordinates of the real sources completely removes the contribution to the signal due to angular clustering. This procedure could therefore underestimate the true correlation length. 
We try to quantify this effect by considering a random sample simulated according to the XMM-COSMOS sensitivity maps (see e.g. Miyaji et al. 2007; and Cappelluti et al. 2007). Briefly, each simulated source is extracted from a reference input $\log N-\log S$, placed at random in the XMM-COSMOS field, and kept in the random sample if its flux is above the sensitivity map value at that position. It is evident that this method is producing a random sample that only accounts for the varying $\mathrm{X}$-ray sensitivity along the COSMOS field, but does not account for the positional biases related to the optical follow-up program. The result of this test is that the measured correlation length increases by $\sim 15 \%$ with respect to the former case.

An additional test was performed prompted by the X-ray flux distribution of objects with spectroscopic redshift being different from that of the total XMM sample. In particular, the fraction of objects with spectroscopic redshift Frac is constant (about 70\%) for X-ray fluxes $f_{0.5-2 \mathrm{keV}}>1.6 \times 10^{-14} \mathrm{erg} \mathrm{cm}^{-2} \mathrm{~s}^{-1}$, while it decreases towards fainter fluxes, reaching 0.0 at $f_{0.5-2} \mathrm{keV}<$ $7 \times 10^{-16}$. Objects with spectroscopic redshifts may therefore undersample the regions of maximum X-ray sensitivity, in which the X-ray source density is higher, producing a more regular distribution on the sky than the total XMM sample. We therefore created a new random sample by first placing sources on the field according to the X-ray sensitivity map as discussed in the previous paragraph and then keeping only a fraction of them, with a flux-dependent "keeping" probability given by the observed relation Frac vs. flux described above. When computing the projected correlation function using this new random sample, we find a result similar to what was obtained in the previous test, i.e. a $\sim 16 \%$ higher $r_{0}$ value than obtained when placing random objects exactly at the coordinates of real objects.

Again, this new random sample unfortunately also does not fully account for the positional biases related to the optical follow-up programs. Indeed, given the very complex optical follow-up that combines results from different programs, it is impossible for us to estimate the correct selection function of our sample, but it is likely that the selection of the masks used for optical spectroscopy, which cover the COSMOS field unevenly, leaving some patches of the field poorly covered, while covering other patches rather extensively, is causing the main positional bias. This can be for instance appreciated in Fig. 1, in which 3 of the 4 inner circles representing Magellan IMACS pointings (16 pointings in total) have a higher density of objects with spectroscopic redshift (cyan dots) than all the remaining pointings. We therefore believe that the systematic upward shift of $15-16 \%$ in $r_{0}$ that we obtained with these tests is likely to be an upper limit. Also, we note that, when performing error analysis considering bootstrap errors (see next paragraph), a difference of $15-16 \%$ is within the total error budget. Given this limited difference, we are confident that our results are not strongly affected by the method used to generate the random source sample.

While many of the correlation function estimators used in the literature have a variance substantially larger than Poisson (because source pairs in general are not independent, i.e. the same objects appear in more than one pair), the estimator used here was shown to have a nearly Poissonian variance (Landy \& Szalay 1993). It has, however, to be noted that the Landy \& Szalay (1993) estimator was originally tested in the approximation of weak clustering, so that Poisson errorbars may in our case underestimate the true uncertainties. Bootstrap resampling has often been used to estimate the uncertainties in the correlation function best-fit parameters (e.g. Mo et al. 1992), but this technique may return an overestimate of the real uncertainties (Fisher et al. 1994). We tested bootstrap errors by randomly extracting
100 samples of 538 sources each from our total sample, allowing for repetitions. The rms in the distribution of the best-fit correlation lengths and slopes is a factor of $\sim 2.8$ and $\sim 2$ greater than the Poisson errorbars, respectively. In the following we simply quote $r_{0}$ and $\gamma$, together with their $1 \sigma$ Poisson errors, bearing in mind that the most likely uncertainty is about a factor of 2 higher.

\section{Results}

We first measured the projected correlation function $w\left(r_{\mathrm{p}}\right)$ of all the 538 spectroscopically identified sources in our XMM-COSMOS reference sample $\left(I_{\mathrm{AB}}<23\right.$ and $\left.z=0.2-3\right)$ regardless of their optical classification. The correlation function was measured on projected scales $r_{\mathrm{p}}=0.3-40 h^{-1} \mathrm{Mpc}$. Here and in the following samples, a simple power law is fit to the data, using standard $\chi^{2}$ minimization techniques to get the best-fit parameters. The best-fit correlation length and slope are found to be $r_{0}=8.65 \pm 0.45 h^{-1} \mathrm{Mpc} \gamma=1.88 \pm 0.07$, respectively. Based on the error on $r_{0}$ from this two-parameter fit, we estimate the clustering signal to be detected at $\gtrsim 18 \sigma$ level, which is the most significant clustering measurement to date for X-ray selected AGN. The measured correlation length appears to be in good agreement with what is estimated by Miyaji et al. (2007) on the first pass on XMM-COSMOS through angular clustering and Limber's inversion. As shown in Fig. 6a, a signal excess above the power-law fit is observed in the scale range $r_{\mathrm{p}} \sim 5-15 h^{-1} \mathrm{Mpc}$, which deserves further investigation. As shown in Fig. 4, a significant fraction of XMM-COSMOS sources are located within a large-scale structure at $z \sim 0.36$ : overall, 42 objects are located at $z=0.34-0.38$, with sky coordinates distributed all over the $2 \mathrm{deg}^{2}$ field $^{4}$. We verified that, when sources in the $z=0.34-0.38$ redshift range are excluded, the signal excess at $r_{\mathrm{p}} \sim 5-15 h^{-1} \mathrm{Mpc}$ actually disappears ${ }^{5}$ (see Fig. 6b). This has the effect of reducing the correlation length to $r_{0}=6.1 \pm 0.8$ (see Table 1). We also verified the effects of restricting the redshift interval around the peak of the redshift distribution and computing $w\left(r_{\mathrm{p}}\right)$ in that interval. For XMM-COSMOS AGN in the range $z=0.4-1.6$, we found a correlation length of $r_{0} \sim 5.2 \pm 1.0 h^{-1} \mathrm{Mpc}$ (see Table 1). Similar results are found when the redshift interval is restricted to $z=0.5-1.5$.

We are repeating our angular auto-correlation function analysis using the full three-year data (55 pointings instead of the first-year 23 pointings used in Miyaji et al. 2007). We plan to use the accurate $(\approx 2 \%)$ photometric redshifts presented by Salvato et al. (2009) to calculate the angular correlation function of XMM AGNs selected by redshift. Preliminary results show $r_{0}=9.7 \pm 2.2 h^{-1} \mathrm{Mpc}$ for the $z>0$ sample, which is fully consistent with the measurement presented in Miyaji et al. (2007), while the correlation length is reduced to $r_{0}=8.9 \pm 2.5 h^{-1} \mathrm{Mpc}$ ( $1 \sigma$ errors) for the sample obtained by excluding objects in the redshift range $z=0.34-0.36$. These values are consistent within the errors with those obtained with the present analysis, despite being systematically higher. The angular analysis results, however, still vary with the angular scale range used for fitting, as well as with error estimation methods. A more detailed

\footnotetext{
${ }_{4}$ Another $\sim 25 \mathrm{X}$-ray selected objects belonging to this structure are found using photometric redshifts (see Fig. 3 and Salvato et al. 2009).

5 We note that, because of this excess due to the $z \sim 0.36$ structure, a simple power law is a very poor fit to the $w\left(r_{\mathrm{p}}\right)$ of our full reference sample and of the $z<1$ AGN subsample (see Sect. 5.3 and Table 1). In contrast, a simple power law provides statistically acceptable fits for all the other subsamples analyzed in this work.
} 

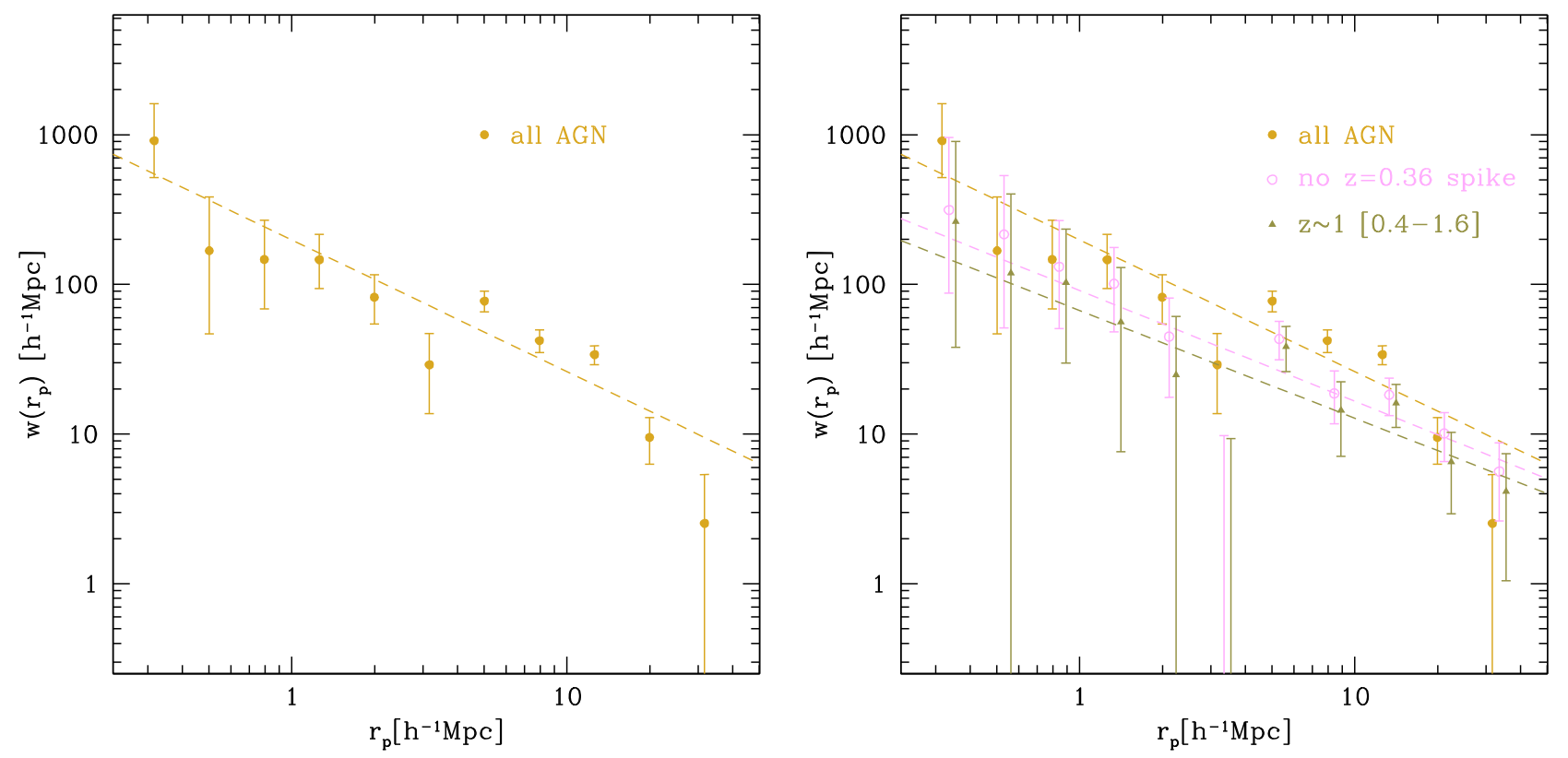

Fig. 6. Left panel: projected correlation function for the XMM-COSMOS AGN reference sample (538 objects with $z=0.2-3.0$ and $I_{\mathrm{AB}}<23$, see Table 1). In this and in the following panel, errors are $1 \sigma$ Poisson confidence intervals and the best-fit power law is shown as a dashed line. Right panel: projected correlation function of the full sample computed including (open circles) and excluding (filled circle) the 42 objects in the large-scale structure at $z \sim 0.36$ : it is evident that the $z \sim 0.36$ structure is largely responsible for the signal excess on scales $r_{\mathrm{p}} \sim 5-15 h^{-1}$ Mpc. The projected correlation function for the $z=0.4-1.6$ sample is also shown (filled triangles). For display purposes, the $w\left(r_{\mathrm{p}}\right)$ datapoints of the different samples have been slightly shifted on the $r_{\mathrm{p}}$ axis.

discussion of the comparison of the results presented in this paper with those obtained through the study of the angular autocorrelation function and cross-correlation function with galaxies will be presented in a future paper (Miyaji et al., in prep.).

\subsection{AGN clustering as a function of optical type}

It is interesting to investigate the projected correlation function for different source subsamples. For each subsample we placed the sources of the random sample only at the positions of the sources in that subsample. The projected correlation function was then fitted both leaving the slope free and fixing it to $\gamma=1.8$, the standard value measured in most galaxy samples, which is also similar to the slope of 1.88 measured for the total XMM-COSMOS sample. Fixing the slope allows a more direct comparison between the correlation lengths of the different subsamples when a two-parameter fit is poorly constrained.

We investigated the clustering properties of sources optically classified as broad line AGN (BLAGN) or non-BLAGN. The class of non-BLAGN is admittedly a mixed bag, which may include obscured AGN, weak unobscured AGN, whose optical emission is diluted by the host galaxy light, and normal galaxies. The cut at $z>0.2$ (roughly corresponding to $L_{0.5-2}>10^{41.5} \mathrm{erg} \mathrm{s}^{-1}$ ), however, should guarantee that the nonBLAGN sample is mostly populated by AGN in which the absence of broad optical lines is solely due to nuclear obscuration. Therefore, investigating the clustering properties of BLAGN and non-BLAGN should be a proxy to investigate the clustering properties of unobscured vs. obscured AGN. For the sample of 305 BLAGN, we measured a correlation correlation length of $r_{0} \sim 7.7 \pm 0.9 h^{-1} \mathrm{Mpc}$ and a slope of $\gamma \sim 2.0 \pm 0.2$, while for the 229 non-BLAGN we measured a similar correlation length, $r_{0} \sim 7.0 \pm 1.0 \mathrm{~h}^{-1} \mathrm{Mpc}$, and a somewhat flatter slope $\gamma \sim 1.6 \pm 0.1$. The projected correlation functions of BLAGN and non-BLAGN AGN are shown in Fig. 7 (upper panel). It should be noted that a proper comparison between the clustering properties of BLAGN and non-BLAGN should take possible redshift effects into account, since BLAGN are generally observed at higher redshift than non-BLAGN (median $z=1.5$ vs. $z=0.7$, see Table 1). In principle, the correlation length of a given AGN and galaxy population is expected to change with redshift, being intimately related to the evolution of the hosting dark matter halos, and one should therefore compare source populations at the same redshift to establish whether they reside in the same environment or not. We will return to this in the discussion.

\subsection{AGN clustering as a function of $X$-ray absorption}

To investigate the clustering properties of obscured vs. unobscured AGN further, we also considered the column density measurements obtained from the spectral analysis of XMM data. We considered here the measurements performed by Mainieri et al. (2007 and in prep.), who performed X-ray spectral fits for those objects with more than 100 counts in the $0.3-10 \mathrm{keV}$ band and found absorption in excess of the Galactic value in about $25 \%$ of their sample (see details in Mainieri et al. 2007). We considered here only objects with more than 200 counts in the $0.3-10 \mathrm{keV}$ band, for which the determination of the column density is more reliable. By matching the Mainieri et al. objects with more than 200 counts with our reference sample, we end up with 290 objects for which a column density has been estimated: 70 of these do show absorption in excess of the galactic value. We note that 21 out of the 190 broad line AGN with measured column density have absorption in excess of the Galactic value, which is consistent with the $10 \%$ fraction of $\mathrm{X}$-ray absorbed broad line AGN found in other X-ray selected samples (see e.g. Tozzi et al. 2006; Brusa et al. 2003). A few words of caution should, however, be spent on these sources. First, simulations run on input X-ray unabsorbed spectra show that, especially for sources at high redshift and relatively low photon 
Table 1. Summary of best-fit clustering parameters.

\begin{tabular}{lccccccc}
\hline \hline Sample & $N^{a}$ & $z$ range & $z^{b}$ & $\log L_{0.5-10}^{c}$ & $\begin{array}{c}r_{0} \\
{\left[h^{-1} \mathrm{Mpc}\right]}\end{array}$ & $\gamma$ & $\begin{array}{c}r_{0}(\gamma=1.8) \\
{\left[h^{-1} \mathrm{Mpc}\right]}\end{array}$ \\
\hline All AGN & 538 & $0.2-3.0$ & 0.98 & 43.8 & $8.65_{-0.48}^{+0.41}$ & $1.88_{-0.07}^{+0.06}$ & $8.39_{-0.39}^{+0.41}$ \\
No $z=0.36$ spike & 496 & $0.2-3.0$ & 1.03 & 43.9 & $6.12_{-0.89}^{+0.64}$ & $1.74_{-0.14}^{+0.13}$ & $6.32_{-0.49}^{+0.53}$ \\
$z \sim 1$ AGN & 349 & $0.4-1.6$ & 0.94 & 43.7 & $5.17_{-1.14}^{+0.80}$ & $1.72_{-0.18}^{+0.17}$ & $5.44_{-0.58}^{+0.62}$ \\
BLAGN & 305 & $0.2-3.0$ & 1.45 & 44.3 & $7.66_{-1.04}^{+0.81}$ & $1.98_{-0.18}^{+0.17}$ & $7.03_{-0.89}^{+0.96}$ \\
non-BLAGN & 229 & $0.2-1.3$ & 0.70 & 43.2 & $7.03_{-1.18}^{+0.87}$ & $1.60_{-0.14}^{+0.13}$ & $7.75_{-0.59}^{+0.62}$ \\
HR $<-0.2$ & 428 & $0.2-3.0$ & 1.15 & 44.0 & $9.56_{-0.45}^{+0.42}$ & $1.98_{-0.07}^{+0.08}$ & $9.05_{-0.45}^{+0.48}$ \\
HR $<-0.2, z<1.3$ & 250 & $0.2-1.3$ & 0.79 & 43.5 & $9.96_{-0.50}^{+0.48}$ & $1.93_{-0.07}^{+0.07}$ & $9.68_{-0.50}^{+0.48}$ \\
HR $<-0.2$, no spike & 218 & $0.2-1.3$ & 0.79 & 43.5 & $6.72_{-0.88}^{+0.72}$ & $1.87_{-0.18}^{+0.18}$ & $6.56_{-0.68}^{+0.75}$ \\
HR $\geq-0.2$ & 102 & $0.2-1.3$ & 0.73 & 43.4 & $5.07_{-1.73}^{+1.58}$ & $2.38_{-0.51}^{+0.85}$ & $4.97_{-1.49}^{+1.93}$ \\
$z<1$ & 276 & $0.2-1.0$ & 0.68 & 43.3 & $7.97_{-0.52}^{+0.48}$ & $1.80_{-0.08}^{+0.08}$ & $7.97_{-0.41}^{+0.43}$ \\
$z<1$ no spike & 234 & $0.2-1.0$ & 0.73 & 43.4 & $5.16_{-0.99}^{+0.71}$ & $1.81_{-0.21}^{+0.20}$ & $5.14_{-0.61}^{+0.66}$ \\
$z>1$ & 262 & $1.0-3.0$ & 1.53 & 44.3 & $6.68_{-1.98}^{+1.19}$ & $1.86_{-0.30}^{+0.27}$ & $6.40_{-0.86}^{+0.94}$ \\
\hline
\end{tabular}

${ }^{a}$ Number of objects in each sample. ${ }^{b}$ Median redshift. ${ }^{c}$ Median X-ray luminosity in the $0.5-10 \mathrm{keV}$ band in units of erg s ${ }^{-1}$. Errors are $1 \sigma$ Poisson confidence levels. Bootstrap errors are a factor of $\sim 2$ larger.

statistics, the spectral fit may return spurious positive values for the absorption (see e.g. Tozzi et al. 2006). Second, the fraction of X-ray absorbed sources might be related to the significance threshold used to assess the presence of X-ray absorption. In particular, Mainieri et al. consider an X-ray source as absorbed if the addition of a photoelectric cut off in the spectral fit improves it at a level of more than $90 \%$ as assessed by an F-test. One would then expect that, in about 7 out of 70 absorbed sources (either BLAGN or non-BLAGN), the measured column density is spurious. At any rate, results do not change significantly if we include those 21 candidate X-ray absorbed BLAGN in the total X-ray absorbed sample or not.

We first verified that the projected correlation function of the 290 objects with X-ray spectroscopy is consistent with that of our full reference sample and then tried to measure the projected correlation function for absorbed and unabsorbed sources separately. Unfortunately, the small number statistics prevent us from getting a significant clustering signal for the $70 \mathrm{X}$-ray absorbed AGN, while for the 220 X-ray unabsorbed objects we found a correlation length of $r_{0}=10.6 \pm 0.9$, somewhat higher than that measured for the subsample of BLAGN. When restricting the analysis to the redshift range $z=0.4-1.6$, i.e. around the peak of the redshift distribution, which also excludes the redshift structure at $z=0.36$, the correlation length of the X-ray unabsorbed objects decreases to $r_{0}=6.2 \pm 1.4$, consistent with what is measured for the full reference sample in the same redshift interval.

To overcome the limitations due to the small size of the sample of objects with reliable $N_{\mathrm{H}}$ measurements, we tried to calibrate a relation between the X-ray column density $N_{\mathrm{H}}$ and the hardness ratio (HR), defined as the difference between the source X-ray photons detected in the $2-10 \mathrm{keV}$ band and those detected in the $0.5-2 \mathrm{keV}$ band, normalized to the sum of the photons in the two bands ${ }^{6}$. The distribution of the column density vs. hardness ratio for objects with more than 200 counts is shown in Fig. 8 (left panel): most of the objects with HR $>-0.2$

\footnotetext{
${ }^{6}$ Upper (lower) limits to the hardness ratio for objects that are not detected in the soft (hard) band have been computed assigning 40 counts in the non-detection band to each source. The distribution of the counts of the detected sources indeed shows a turnover at this value, which therefore appears as the average count threshold for source detection.
}

do show absorption in excess of $\log N_{\mathrm{H}}=21.6$, therefore we adopt a rough threshold at $\mathrm{HR}=-0.2$ to divide X-ray absorbed from X-ray unabsorbed AGN (see also Hasinger 2008; and Fig. 11 in Mainieri et al. 2007). The HR distribution as a function of redshift for the 538 objects in the reference sample is shown in Fig. 8 (right panel). Most BLAGN fall below the $\mathrm{HR}=-0.2$ line, while non-BLAGN AGN do show higher HR values on average. The poorly populated upper-right corner of the figure, i.e. the high-HR - high- $z$ region, suffers from obvious selection effects due to i) the bias against faint (distant and absorbed) magnitude targets in the optical spectroscopy follow-up (see also Fig. 4 in Brusa et al. 2007) and ii) the K-correction effects that make high-redshift absorbed spectra to appear softer in the X-ray bandpass (i.e. lower HR values). It is noted that BLAGN form a sort of horizontal sequence at $\mathrm{HR} \sim-0.5$, which is indeed the hardness ratio value expected by a canonical power law spectrum with photon index $\Gamma=1.7$ and no absorption. Since we consider all objects with HR $<-0.2$ as unabsorbed sources, the adopted cut conservatively accounts for any dispersion in the photon index distribution of BLAGN. We measured $w\left(r_{\mathrm{p}}\right)$ for absorbed and unabsorbed objects separately. At projected scales below $r_{\mathrm{p}} \sim 1 h^{-1} \mathrm{Mpc}$, absorbed and unabsorbed AGN are similarly correlated, while absorbed AGN appear less correlated on larger scales. This results in absorbed AGN formally having a lower correlation length $\left(r_{0}=5.1 \pm 1.7\right.$ vs. $\left.9.6 \pm 0.4 h^{-1} \mathrm{Mpc}\right)$ and a steeper slope ( $\gamma=2.4 \pm 0.7$ vs. $\left.2.0 \pm 0.1\right)$ than unabsorbed objects. The projected correlation function for unabsorbed AGN does not change significantly if we restrict the analysis to the redshift range $0.2-1.3$, i.e. the same range as used for absorbed AGN (see Table 1). The projected correlation function of unabsorbed and absorbed AGN in the same $z=0.2-1.3$ redshift interval are shown in Fig. 7 (middle panel). The larger correlation length measured for unabsorbed objects is essentially due to most objects in the $z=0.36$ structure having $\mathrm{HR}<-0.2$ (see Fig. 8, right panel). Indeed, when removing this structure, the correlation length for X-ray unabsorbed AGN decreases to $r_{0}=6.7 \pm 0.8$ (see Fig. 7 , middle panel), which, given the large errorbars, is not significantly different from that of X-ray absorbed objects. To summarize, from our analysis we 


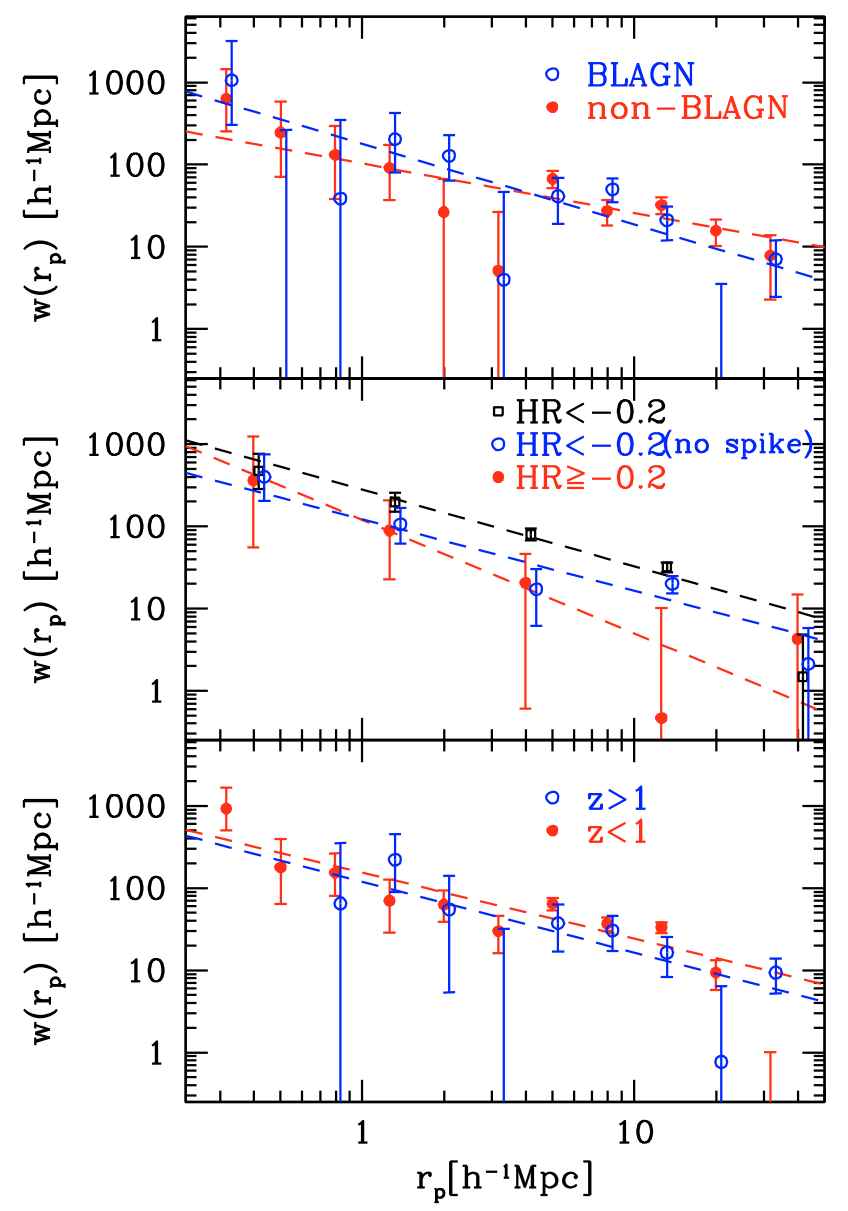

Fig. 7. Projected correlation function for different XMM-COSMOS AGN subsamples. Upper panel: broad line AGN (open circles) vs. non-broad line AGN (filled circles). Middle panel: X-ray unabsorbed $(\mathrm{HR}<-0.2)$ AGN (open squares) vs. X-ray absorbed $(\mathrm{HR} \geq-0.2)$ AGN (filled circles). Since the $z=0.36$ redshift structure is mainly populated by X-ray unabsorbed objects (see Fig. 8 right panel), we also plotted the projected correlation function obtained for X-ray unabsorbed AGN after removing objects at $z \sim 0.36$ (open circles). Lower panel: AGN at $z>1$ (open circles) vs. AGN at $z<1$ (filled circles). In all panels errors are $1 \sigma$ Poisson confidence intervals and the best fit power laws are shown as dashed lines.

cannot claim that X-ray absorbed and X-ray unabsorbed AGN possess different clustering properties.

\subsection{AGN clustering as a function of redshift}

The study of AGN clustering as a function of redshift provides several pieces of information about the formation and evolution of the AGN population.

Because of the limited sample size, we simply split the XMM-COSMOS AGN sample in two subsamples of objects below and above redshift 1 . The correlation functions of the 276 AGN at $z<1$ and of the 262 AGN at $z>1$ are shown in Fig. 7 (lower panel). The best-fit correlation parameters for objects at $z<1$ are found to be $r_{0} \sim 8.0 \pm 0.5 h^{-1} \mathrm{Mpc}$ and $\gamma \sim 1.8 \pm 0.1$, while for objects at $z>1$ the best-fit parameters are $r_{0} \sim 6.7 \pm 1.6 h^{-1} \mathrm{Mpc}$ and $\gamma \sim 1.9 \pm 0.3$. When removing the $z=0.36$ structure, the correlation length of objects at $z<1$ decreases to $r_{0} \sim 5.2 \pm 0.8 h^{-1} \mathrm{Mpc}$ (see Table 1). In Sect. 6.3. we discuss the correlation lengths of the various XMM-COSMOS redshift subsamples as compared to those of other optical and $\mathrm{X}$-ray selected samples at different redshifts.

\subsection{AGN clustering as a function of luminosity}

We finally investigated the dependence of the AGN clustering parameters on the X-ray luminosity, since this may reveal whether objects shining with different luminosities reside in dark matter halos with different masses, hence constraining the distribution of the AGN Eddington ratios (see e.g. Lidz et al. 2006; Marulli et al. 2009, and the discussion in Sect. 6.3). Again, because of the limited size of the sample, we simply divided it into two almost equally populated subsamples, the dividing line being at $L_{0.5-10 \mathrm{keV}}=10^{44} \mathrm{erg} \mathrm{s}^{-1}$ (corresponding to $L_{0.5-2 \mathrm{keV}} \sim$ $10^{43.5} \mathrm{erg} \mathrm{s}^{-1}$ for a typical AGN X-ray spectrum). As shown in Fig. 2, splitting the sample at this luminosity is almost equal to splitting the sample at a redshift of $z \sim 1$. Indeed, when computing the clustering parameters of the higher (lower) luminosity sample, these are very similar to the $z>1(z<1)$ sample, and therefore are not reported here.

\section{Discussion}

\subsection{Comparison with other $z \sim 1 A G N$ and galaxy samples}

The galaxy and AGN census in the $z \sim 1$ Universe has been recently enlarged by a number of surveys with different areas and sensitivities, which allowed investigation of the spatial distribution of different populations. The comparison between the clustering properties of AGN and galaxies allows to first approximation to infer which galaxy population is hosting any given AGN population, under the simple hypothesis that AGN activity at a given redshift is randomly sampling the host galaxy population. The comparison between $z \sim 1$ AGN samples obtained from surveys with different sensitivities may also reveal any dependence of AGN clustering on luminosity. As far as X-ray selected AGN are concerned, a correlation length of $r_{0}=5.7_{-1.5}^{+0.8} h^{-1} \mathrm{Mpc}$ has been measured for $\sim 230$ objects in the $0.4 \mathrm{deg}^{2}$ CLASXS survey (Yang et al. 2006). Objects in the CLASXS have redshift and luminosity distributions very similar to those of our sample, and therefore they should trace the AGN population sampled by XMM-COSMOS almost exactly. To check whether the different techniques used for the clustering analysis may introduce significant differences, we analyzed the CLASXS sample using the same techniques as were used in this work finding best-fit clustering parameters in very good agreement with the Yang et al. (2006) values. The difference between the $r_{0}$ values measured in XMM-COSMOS and in CLASXS full samples (8.6 vs. $5.7 h^{-1} \mathrm{Mpc}$, respectively) therefore appears to be inherent to the two fields considered. While a prominent redshift spike is observed at $z=0.36$ in XMM-COSMOS, no such similar structures are found in the CLASXS field. Indeed, when removing the structure at $z=0.36$, the correlation length of XMM-COSMOS AGN decreases to $\sim 6.3 h^{-1} \mathrm{Mpc}$, in good agreement with the value measured in CLASXS. Moreover, when restricting the analysis to XMM-COSMOS AGN in the redshift range $z=0.4-1.6$, the correlation length $\left(\sim 5.2 h^{-1} \mathrm{Mpc}\right)$ is very similar to what is measured in CLASXS. One therefore may wonder about the frequency with which prominent largescale structures are sampled in X-ray surveys of different sky areas, i.e. the effects of cosmic variance. Indeed, based on simulated galaxy mock catalogs over $2 \mathrm{deg}^{2}$ fields (Kitzbichler \& White 2006), some evidence exists that the COSMOS field has some excess of structures with respect to the average. 


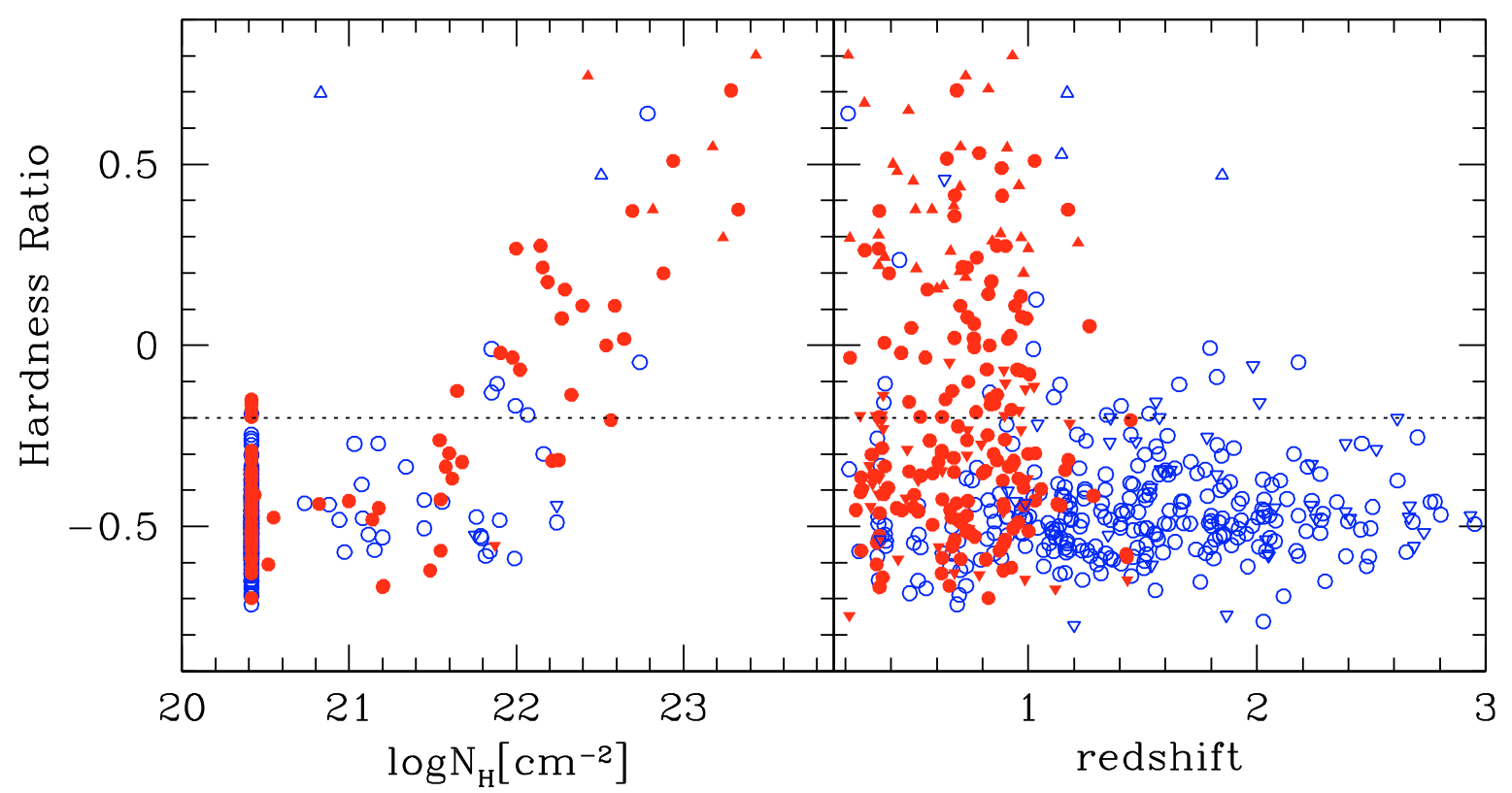

Fig. 8. Left panel. Column density $N_{\mathrm{H}}$ vs. hardness ratio distribution for the 290 objects in our reference sample with more than 200 photons in the 0.5-10 keV band, for which an accurate $N_{\mathrm{H}}$ measurement can be performed through X-ray spectral fitting. Objects that do not show absorption have been plotted at $\log N_{\mathrm{H}}=20.4$, i.e. at the Galactic value. Broad line AGN and non-broad line AGN are shown as blue open and red filled symbols, respectively. Circles refer to objects detected in both soft and hard X-ray bands, for which an HR value is directly measured. Upward (downward) pointing triangles represent lower (upper) limits to the hardness ratios. It is evident that most objects above the dotted line at HR $=-0.2$ are absorbed by column densities $\log N_{\mathrm{H}}>21$.6. Right panel. Hardness ratio vs. redshift plot for the 538 sources in our reference sample. Symbols are as in the left panel. The dotted line at $\mathrm{HR}=-0.2$ marks the adopted dividing line between X-ray absorbed and unabsorbed AGN.

The correlation length of XMM-COSMOS AGN can be compared to that of different galaxy populations at $z \sim 1$. Coil et al. (2004) find $r_{0}=3.2 \pm 0.5 h^{-1} \mathrm{Mpc}$ for emission line galaxies in the DEEP2 survey, while Meneux et al. (2006) find $r_{0}=2.5 \pm 0.4 h^{-1} \mathrm{Mpc}$ for star-forming blue galaxies in the Vimos-VLT Deep Survey (VVDS, Le Fevre et al. 2004). The populations of red absorption-line galaxies in the same surveys have instead larger correlation lengths: $r_{0} \sim 6.6 h^{-1} \mathrm{Mpc}$ for absorption line galaxies in the DEEP2 (Coil et al. 2004) and $r_{0}=4.8 \pm 0.9 h^{-1} \mathrm{Mpc}$ for red, early type galaxies in the VVDS (Meneux et al. 2006). Recently, a correlation length as large as $r_{0}=5.1 \pm 0.8 h^{-1} \mathrm{Mpc}$ has been measured for luminous infrared galaxies (LIRG, $L_{\mathrm{IR}}>10^{11} L_{\odot}$ ) at $z \sim 0.8$, which are forming stars at high rates ( $S F R>17 M_{\odot} \mathrm{yr}^{-1}$; see Gilli et al. 2007b). Since at $z \sim 1$ star formation is closely related to galaxy mass (Noeske et al. 2007; Elbaz et al. 2007), even LIRGs, as well as $z \sim 1$ early type galaxies, are massive objects with stellar mass $M_{\star} \gtrsim 3 \times 10^{10} M_{\odot}$. The fact that XMM-COSMOS AGN show similar correlation length to these systems (see Fig. 9), suggests that, similar to what is observed at $z=0$ (Kauffmann et al. 2004) and at $z \sim 2$ (Daddi et al. 2007), at $z \sim 1$ nuclear activity is hosted by the more massive galaxies (see also Georgakakis et al. 2007). This is in good agreement with the analysis by Silverman et al. (2008b), who investigated the occurrence of nuclear activity on a sample of $\sim 8000$ galaxies selected from the zCOSMOS spectroscopic catalog, finding that the fraction of galaxies hosting an AGN increases towards large stellar masses at $z \lesssim 1.0$. In particular, most $(\sim 80 \%)$ AGN at $z \sim 1$ reside in galaxies with stellar mass $M_{\star}>3 \times 10^{10} M_{\odot}$, in agreement with the conclusions from our clustering analysis.

\subsection{The connection with dark matter halos}

While on small scales, comparable to the dimensions of dark matter halos, AGN and galaxy clustering are difficult to predict because of merging and interactions that can trigger a number of physical processes, on larger scales (e.g., $>1 h^{-1} \mathrm{Mpc}$ ), where interactions are rare, the AGN correlation function should follow that of the hosting dark matter halos.

An interesting consequence is that one can estimate the masses of the typical halos hosting an AGN population by simply comparing their clustering level. According to the standard $\Lambda \mathrm{CDM}$ hierarchical scenario, dark matter halos of different mass cluster differently, with the more massive halos more clustered for any given epoch, and it is straightforward to compute the correlation function for halos above a given mass threshold. It is worth noting that, since less massive halos are more abundant, the correlation function of halos above a given mass threshold is very similar to the clustering of halos with mass close to that threshold. Also, it is important to note that, as far as our measurements are concerned, the best-fit clustering parameters are obtained from datapoints mostly on large scales $\left(r_{\mathrm{p}}>1 h^{-1} \mathrm{Mpc}\right.$; see Fig. 6). Therefore the measured $r_{0}$ and $\gamma$ values are essentially due to the clustering signal on large scales, where the AGN correlation function follows that of the dark matter, allowing a meaningful comparison with the clustering expected for dark matter halos.

We considered the dark matter halo catalogs available for the Millennium simulation ${ }^{7}$ (Springel et al. 2005). Halo catalogs are available at different time steps along the simulation. Here we considered those at $z \sim 1$ (parameter stepnum=41 in the simulation). In total there are about $1.6 \times 10^{7}$ halos with mass above $10^{10} h^{-1} M_{\odot}$ in a cubic volume of $500 h^{-1} \mathrm{Mpc}$ on a side. We computed the correlation function and the space density

\footnotetext{
${ }^{7}$ See http://www .mpa-garching.mpg.de/millennium.
} 


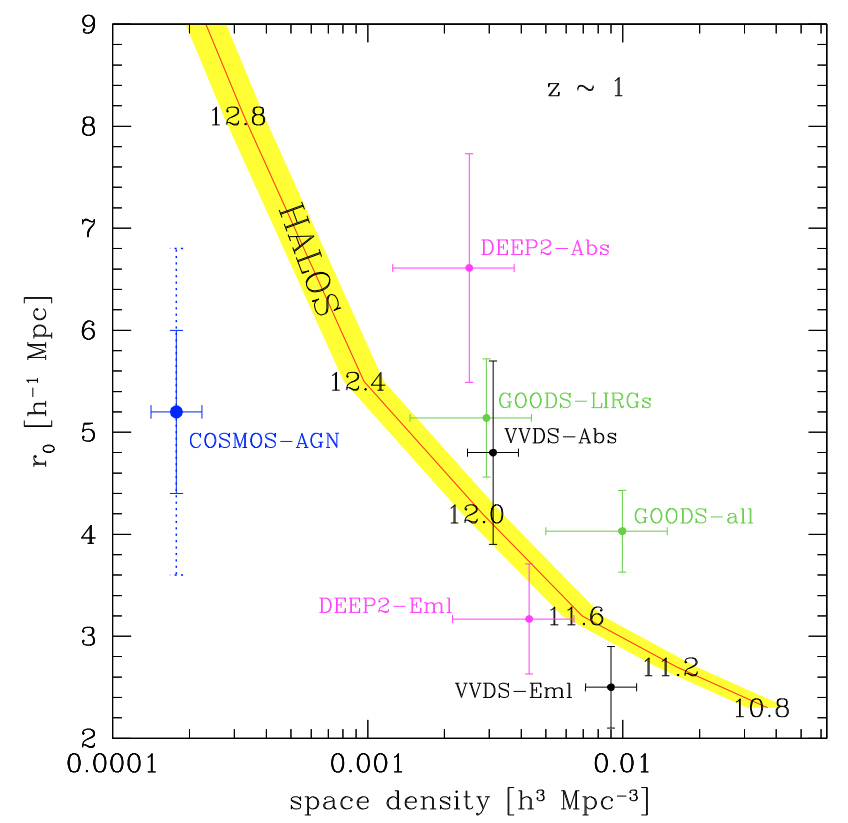

Fig. 9. Space density vs. correlation length for the XMM-COSMOS AGN compared to that of other AGN and galaxy populations at $z \sim 1$, as labeled. The trend for the Millennium dark matter halos at $z \sim 1$ above different mass thresholds is also shown as a shaded region. More massive halos (log of the threshold mass is labeled) are less abundant and more clustered than less massive ones. The XMM-COSMOS AGN datapoint (big filled circle) refers to the $z=0.4-1.6$ sample. Solid and dotted errorbars correspond to Poissonian and bootstrap uncertainties, respectively.

of halos above 7 mass thresholds ranging from $\log \left(M / M_{\odot}\right)=$ 10.8 to 13.2 in steps of 0.4 . Here we use as halo mass estimator the simulation parameterm_Crit200, defined as the mass within the radius where the integrated halo overdensity is 200 times the critical density of the simulation. The halo correlation length was estimated by fitting with a power law the halo correlation functions on scales above $r=1 h^{-1} \mathrm{Mpc}$. The results are shown in Fig. 9, where it is clear that more massive halos are more clustered and less numerous.

We computed the space density of AGN in XMM-COSMOS as expected from published X-ray luminosity functions (see details in Sect. 6.5) and compared the $r_{0}$ and density values of our population with those of other AGN and galaxy populations at $z \sim 1$ and with those of dark matter halos at $z \sim 1$ as computed above. We considered the XMM-COSMOS AGN in the redshift range $z=0.4-1.6$, i.e. around the peak of the selection function and excluding the redshift structure at $z=0.36$. The comparison is shown in Fig. 9.

By comparing the halo and the galaxy $r_{0}$ values, XMM-COSMOS AGN appear to be hosted by halos with masses $\gtrsim 2.5 \times 10^{12} M_{\odot}$, similar to absorption line galaxies and LIRGs, which indeed show similar correlation lengths. However, while absorption line galaxies and LIRGs appear to be more abundant than the hosting halos (with an average of 2-4 such galaxies per halo), XMM-COSMOS AGN appear to be a factor of $\gtrsim 5$ less abundant than the hosting halos, suggesting that nuclear activity is present in about $\sim 15-20 \%$ of halos of that mass. Considerations about the duty cycle and lifetimes of XMM-COSMOS AGN will be presented in Sect. 6.5.

\subsection{Evolution of AGN clustering}

To investigate the evolution of the AGN clustering properties with redshift we combined the results from XMM-COSMOS with recent findings from other X-ray and optical surveys. When necessary, the results were corrected to the cosmology adopted here. The values of the correlation lengths reported in this section were usually calculated by fixing $\gamma$ to 1.8 , therefore allowing a consistent comparison. When different slopes were measured/adopted, we discuss the case and verify the effects of assuming $\gamma=1.8$. Only results from spatial clustering analysis are considered.

As for the X-ray surveys we considered the results from the ROSAT NEP survey and from the Chandra Msec fields. In the NEP survey, Mullis et al. (2004) found a correlation length of $r_{0} \sim 7.4 \pm 1.8 h^{-1} \mathrm{Mpc}$ on scales of 5-60 $h^{-1} \mathrm{Mpc}$ for source pairs at a median redshift $\bar{z}=0.22$. In the CDFS and CDFN, the correlation length measured by Gilli et al. (2005) is $r_{0}=$ $10.3 \pm 1.7 h^{-1} \mathrm{Mpc}$ and $r_{0}=5.5 \pm 0.6 h^{-1} \mathrm{Mpc}$, respectively. Although the best-fit slopes in the Chandra Msec fields are rather flat $(\gamma=1.3-1.5)$, the best-fit correlation lengths increase by only $\sim 15 \%$ if the slope is fixed to 1.8 (Gilli et al. 2005).

As for optically selected AGN, we considered the results of Croom et al. (2005) based on more than 20000 objects in the final catalog of the $2 \mathrm{dF}$ QSO Redshift Survey (2QZ), where the QSO correlation length is found to increase significantly from $z \sim 1$ to $z \sim 2.5$, and very flat slopes $(\gamma \sim 1.1-1.2)$ have been measured. As discussed by Croom et al. (2005), these flat slopes stem from redshift-space distortions that are relevant when the correlation function is measured down to small scales in redshift rather than in real space. The real-space clustering for the total 2QZ sample has instead been measured by Da Angela et al. (2005) via the projected correlation function. On the same scales as considered by Croom et al. (2005) and when approximating $\xi(r)$ by a single power law, they found that, while the slope of the total 2QZ sample steepens significantly from $\gamma=1.20 \pm 0.10$ to $\gamma=1.85 \pm 0.13$, the correlation length only marginally decreases by $10 \%$ (from $r_{0}=5.5 \pm 0.5 h^{-1} \mathrm{Mpc}$ to $r_{0}=5.0 \pm 0.5 h^{-1} \mathrm{Mpc}$ ). In the following we therefore simply consider the values as measured by Croom et al. (2005) for the 2QZ correlation lengths in different redshift bins. These results are consistent with those obtained by Porciani et al. (2004) using $2 \mathrm{dF}$ QSOs in a narrower redshift range. In the local Universe $(z \sim 0.07)$, the clustering of bright optical QSOs $(B<15 \mathrm{mag})$ has been recently determined by Grazian et al. (2004) by means of the Asiago-ESO/RASS QSO survey (AERQS). These authors measured $r_{0}=8.6 \pm 2.0 h^{-1} \mathrm{Mpc}$ at a median redshift of $z \sim 0.1$ on comoving scales $1-30 h^{-1} \mathrm{Mpc}$ by fixing the correlation slope to $\gamma=1.56$. Given the above considerations for the Chandra Msec fields and the 2QZ and given the rather large uncertainties we have to deal with, we consider the value quoted by Grazian et al. (2004) as if obtained by fixing $\gamma$ to 1.8. All the measurements discussed above are shown in Fig. 10.

Unfortunately, it is not possible to perform a completely unbiased comparison between the various samples because different redshifts generally sample different luminosities, and AGN clustering may be a function of AGN luminosity if the latter correlates with the mass of the hosting dark halo (e.g. Kauffmann \& Haehnelt 2002).

In Fig. 10, sources at $z<0.3$ and $z>2$ appear to be the most clustered ones, and these also correspond to the most luminous AGN. At $z<0.3$ the median $0.5-10 \mathrm{keV}$ luminosity of the AGN in the AERQS and NEP samples is about $\log L_{x}=44.4$ (see Mullis et al. 2004; and Gilli et al. 2005). At $z>2$ the me- 


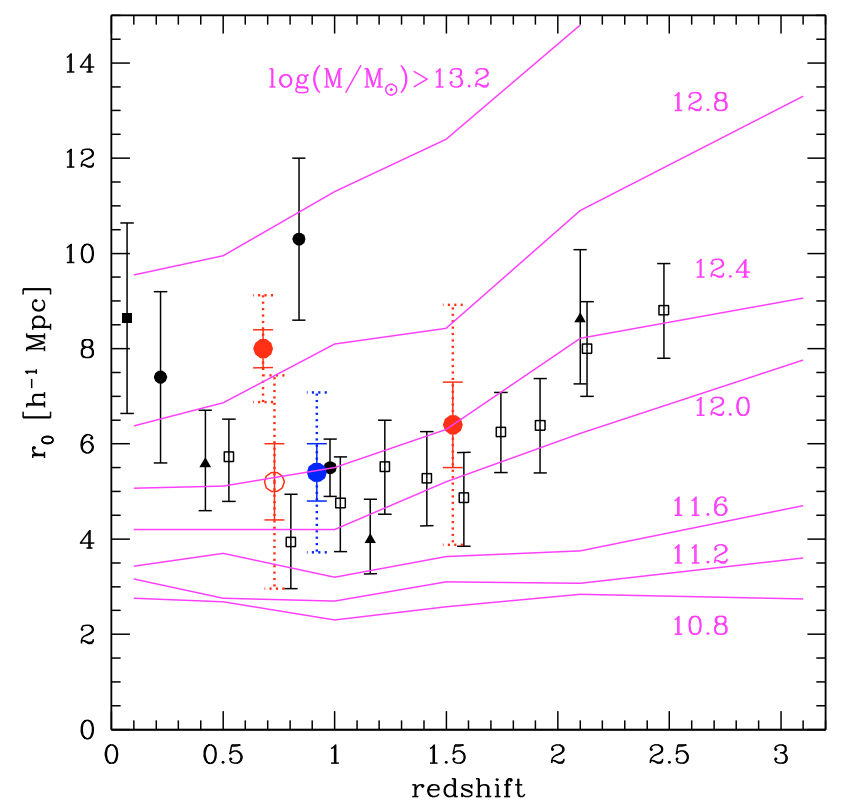

Fig. 10. Correlation length vs. redshift for XMM-COSMOS AGN compared to that of other AGN samples. The big blue filled circle refers to the $z=0.4-1.6$ XMM-COSMOS sample. Big red filled circles refer to the $z<1$ and $z>1$ samples. The big open circle refers to the $z<1$ sample when excluding the $z=0.36$ spike. Solid and dotted errorbars correspond to Poissonian and bootstrap uncertainties, respectively. The filled square at $z \sim 0.1$ refers to the AERQS QSO sample (Grazian et al. 2004). Filled circles at $z \sim 0.2,0.7,0.9$ refer to the NEP (Mullis et al. 2004), CDFS and CDFN samples (Gilli et al. 2005), respectively. Filled triangles refer to the CLASXS sample (see e.g. Yang et al. 2006). Open squares refer to optically selected QSOs in the 2QZ sample (Croom et al. 2005). The solid curves show the correlation length vs. redshift relations expected for dark matter halos in the Millennium simulation above different mass thresholds as labeled.

dian absolute $B$-band luminosity of the 2QZ QSOs corresponds to a median $0.5-10 \mathrm{keV}$ luminosity of $\log L_{x}=44.7$ (assuming a standard QSO SED, e.g. Elvis et al. 2009). The less clustered sources are found at $z \sim 1$, but these have lower luminosities ( $\left.\log L_{x}=43-44\right)$. In general, a clear dependence of clustering amplitude on AGN luminosity has not been observed yet. On the contrary, the available evidence, if any, points towards a similar clustering for sources at the same redshift but with different luminosities. Croom et al. (2005) and Porciani \& Norberg (2006) could not find any significant evidence of luminosity dependent clustering in the 2QZ. From a cross-correlation analysis between galaxies and AGN, Adelberger \& Steidel (2005) claim that AGN at $z \sim 2$ cluster similarly within a 10 mag luminosity range. From a theoretical point of view, one would expect little clustering dependence on the observed AGN luminosity if this is not directly related to the host halo mass; i.e., if, at any given redshift, objects that reside in halos within a narrow mass range have very different luminosities (e.g. Lidz et al. 2006). For instance, even assuming a dependence of black hole (and host galaxy) mass on the hosting halo mass, a wide spread in the distribution in the Eddington ratios would make $\mathrm{BH}$ of similar masses radiate at very different luminosities. Indeed, although the average Eddington ratio of SDSS QSOs has been shown to increase towards high luminosities, the spread in the distribution is wide (McLure \& Dunlop 2004). Moreover, it has been recently suggested (Gavignaud et al. 2008) that the dispersion in the black hole mass-luminosity increases even more for lower AGN luminosities. Finally, in the local Universe, Constantin \&
Vogeley (2006) find that low-luminosity LINERs are more clustered than higher luminosity Seyfert galaxies, showing that the relation between AGN luminosity and clustering may even be reversed for low-luminosity AGN with respect to the expectations based on a monotonically increasing relation between luminosity and black hole mass. Large statistical samples, beyond the reach of the data presented in this work, are needed to firmly establish any dependence of AGN clustering on luminosity (see e.g. Porciani \& Norberg 2006).

Overall, when removing the redshift structure at $z=0.36$, the clustering of XMM-COSMOS AGN appears in good agreement with what is measured for optical and X-ray selected AGN at different redshifts. A larger correlation length is instead found for objects at $z<1$ if the redshift structure is not removed.

To interpret our clustering measurements at different redshifts, we considered the halo catalogs in the Millennium simulation and computed their correlation function above different halo mass thresholds and at different redshifts. We essentially repeated the computation presented in Sect. 6.2 for halos at $z=0.1,0.5,1.0,1.5,2.0$, and 3.0. The $r_{0}$ vs. redshift curves for halos above different mass thresholds are shown in Fig. 10. Both our $z \sim 1$ and $z>1$ AGN samples seem to be hosted by halos with mass above $\log \left(M / M_{\odot}\right)>12.4$. For XMM-COSMOS AGN at $z<1$ the minimum mass of the host halos varies from 12.4 to 12.8 depending on whether the $z=0.36$ structure is excluded or included from the computation of $r_{0}$.

\subsection{Descendants of $z \sim 1 A G N$}

As shown in the previous sections, under simple assumptions, it is possible to use the spatial clustering of an extragalactic source population measured at a given epoch to estimate the typical dark matter halos in which these objects reside. Furthermore, it is also possible to estimate their past and future history by following the halo evolution in the cosmological density field. A useful quantity for such analyses is the bias factor, defined as $b^{2}(r, z, M)=\xi_{\mathrm{A}}(r, z, M) / \xi_{\mathrm{m}}(r, z)$, where $\xi_{\mathrm{A}}(r, z, M)$ and $\xi_{\mathrm{m}}(r, z)$ are the correlation function of the considered AGN or galaxy population and that of dark matter, respectively. In general the bias parameter can be a function of scale $r$, redshift $z$, and object mass $M$. For simplicity we adopt the following definition here:

$b^{2}(z)=\xi_{\mathrm{A}}(8, z) / \xi_{\mathrm{m}}(8, z)$

in which $\xi_{\mathrm{A}}(8, z)$ and $\xi_{\mathrm{m}}(8, z)$, are the galaxy and dark matter correlation functions evaluated at $8 h^{-1} \mathrm{Mpc}$, respectively. The AGN correlation function has been measured directly in this work, while the dark matter correlation function can be estimated using the following relation (e.g., Peebles 1980):

$\xi_{\mathrm{m}}(8, z)=\sigma_{8}^{2}(z) / J_{2}$

where $J_{2}=72 /\left[(3-\gamma)(4-\gamma)(6-\gamma) 2^{\gamma}\right]$, and $\sigma_{8}^{2}(z)$ is the dark matter mass variance in spheres of $8 h^{-1} \mathrm{Mpc}$ comoving radius, which evolves as $\sigma_{8}(z)=\sigma_{8}(0) D(z)$. Also, $D(z)$ is the linear growth factor of perturbations, while $\sigma_{8}(0)$ is the rms dark matter fluctuation at the present time, which we fix to $\sigma_{8}=0.8$ in agreement with the recent results from WMAP3 (Spergel et al. 2007) ${ }^{8}$. While in an Einstein - De Sitter cosmology the linear growth of perturbations is simply described by $D_{\text {EdS }}(z)=(1+z)^{-1}$, the growth of perturbations is slower in a

${ }^{8}$ Only small differences arise if we assume $\sigma_{8}=0.9$ as in the Millennium simulation. The other relevant cosmological parameters assumed in this work are the same as in the Millennium simulation. 


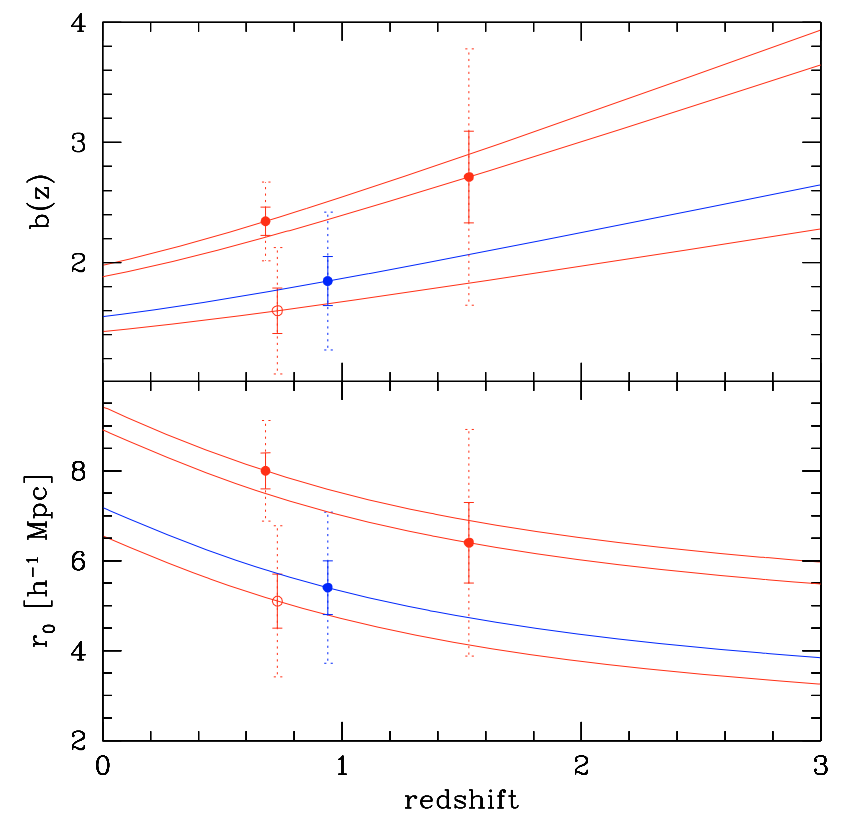

Fig. 11. Expected redshift evolution of the bias and correlation length of different XMM-COSMOS AGN samples according to a conserving scenario. The evolution curves are normalized to the observed datapoints. Blue filled circle: $z=0.4-1.6$ sample. Red circles: $z<1$ and $z>1$ samples. The open circle shows the result for the $z<1$ sample when the $z=0.36$ structure is excluded. Solid and dotted errorbars correspond to Poissonian and bootstrap uncertainties, respectively.

$\Lambda$-dominated cosmology. We consider here the so-called growth suppression factor $g(z)=D(z) / D_{\mathrm{EdS}}(z)$ as approximated analytically by Carroll et al. (1992). The resulting bias for the AGN in the XMM-COSMOS field is $b(1.0)=2.0 \pm 0.2$.

Once the bias of the AGN population at its median redshift has been estimated using the above relations, it is possible to follow the time evolution of the bias with models that rely on simple assumptions. Two popular scenarios that encompass two extreme hypotheses are the conserving model, in which objects do not merge at all (Nusser \& Davis 1994; Fry 1996) and the merging model in which objects merge continuously (e.g. Moscardini et al. 1998). In the first hypothesis the number of objects is conserved in time, and galaxies behave as test particles whose spatial distribution simply evolves with time under the gravitational pull of growing dark matter structures. In the second hypothesis, object merging follows the continuous merging of the hosting dark matter halos, in such a way that only those objects and halos which have just merged are observable at any given epoch.

From an observational point of view, the fraction of galaxies in mergers appears to be a very debated issue. Recent works suggest that close galaxy pairs (merger candidates) are a strong function of redshift, evolving as $(1+z)^{3-4}$ (Kartaltepe et al. 2007; Kampzyck et al. 2007). By extrapolating the current estimates, at $z \sim 2$ about $50 \%$ of luminous galaxies are expected to be found in close pairs/mergers. However, at $z \sim 1$ the fraction of galaxies in close pairs is still $\sim 8 \%$ and decreases to $\sim 0.1 \%$ at $z \sim 0.1$. In the following we will consider the non merging conserving model as a fairly adequate representation of the bias evolution of $z \sim 1$ XMM-COSMOS AGN towards lower redshifts; i.e., it will be used to estimate the likely descendants of XMM-COSMOS AGN. On the contrary, since merging is expected to be significant towards higher redshift, we will not try to estimate their high-z progenitors.
In the galaxy conserving model, the bias evolution can be approximated by

$b(z)=1+[b(0)-1] / D(z)$

where $b(0)$ is the population bias at $z=0$ (Nusser \& Davis 1994; Fry 1996; Moscardini et al. 1998). Once $b(z)$ is determined, the evolution of $\xi_{\mathrm{A}}(8, z)$ and hence of $r_{0}(z)$ can be obtained by inverting Eq. (11). A value of $\gamma \sim 1.8$ for the slope is assumed in the above relations. Little difference arises when using $\gamma=1.9$.

In Fig. 11 we show the evolution of $b(z)$ and $r_{0}(z)$ for various XMM-COSMOS AGN samples, including the $z=0.4-1.6$ sample, the sample at $z>1$ and the sample at $z<1$ with or without the structure at $z=0.36$. By $z=0$ the correlation length of XMM-COSMOS AGN should evolve to $r_{0} \gtrsim 6 h^{-1} \mathrm{Mpc}$, which is typical of passive, early type galaxies in the local Universe (Colless et al. 2001; Zehavi et al. 2004). The correlation slope of the local early type population $\gamma \sim 1.8-2.0$ also appears consistent with that of XMM-COSMOS AGN at $z \sim 1$. In principle, the evolution curves shown in Fig. 11 can also be used to predict which $r_{0}$ value a given XMM-COSMOS subsample should have as a function of redshift, allowing a proper comparison between measurements obtained at different redshifts. Indeed, the correlation length of AGN in the $z>1$ sample (median $z \sim 1.5$ ) is expected to evolve to $r_{0} \sim 7.5 h^{-1} \mathrm{Mpc}$ by $z \sim 0.7$, i.e. the median redshift of the $z<1$ sample, whose correlation length has been measured as varying between 8.0 and $5.2 \mathrm{~h}^{-1} \mathrm{Mpc}$, depending on the inclusion of the $z=0.36$ structure. Given this uncertainty and the large errorbars in Fig. 11, it is still difficult to claim that objects at redshift greater or smaller than 1 are sampling different environments.

\subsection{Estimating the AGN lifetime}

Under simple assumptions it is possible to put limits on the AGN lifetime at any given redshift. Following Martini \& Weinberg (2001), we assumed that the AGN in our sample reside within halos above a given mass threshold and that each halo hosts at most one active AGN at a time. The AGN lifetime $t_{Q}$ can then be estimated with the following relation:

$\Phi(z)=\int_{M_{\min }}^{\infty} \mathrm{d} M \frac{t_{Q}}{t_{\mathrm{H}}(M, z)} n(M, z)$,

where $\Phi(z)$ is the comoving space density of AGN above a given luminosity, $M_{\min }$ the minimum mass of the halos hosting an AGN, $n(M, z)$ the comoving space density of halos of mass $M$ at redshift $z$, and $t_{\mathrm{H}}(M, z)$ is the lifetime of halos of mass $M$ at redshift $z$.

The definition of halo lifetime is somewhat ambiguous since halos are continuously accreting matter. Martini \& Weinberg (2001) defined $t_{\mathrm{H}}(M, z)$ as the median time interval for a halo of mass $\mathrm{M}$ to be incorporated into a halo of mass $2 \mathrm{M}$ and used the extended Press-Schechter formalism to calculate it. To a first approximation $t_{\mathrm{H}}(M, z) \sim t_{U}(z)$, where $t_{U}(z)$ is the Hubble time at redshift $z$. With these approximations Eq. (14) can be rewritten as

$t_{Q}(z)=t_{u}(z) \frac{\Phi(z)}{\Phi_{\mathrm{H}}(z)}$

where $\Phi_{\mathrm{H}}(z)=\int_{M_{\min }}^{\infty} \mathrm{d} M n(M, z)$ is the comoving space density of halo with mass above $M_{\min }$.

Since $M_{\min }$ is known from the comparison between the halo and the AGN correlation length, it is straightforward to estimate $\Phi_{\mathrm{H}}(z)$ from the number of halos with $M>M_{\min }$ within 
the Millennium simulation box. For halos with $M>2.5 \times$ $10^{12} h^{-1} M_{\odot}$, where XMM-COSMOS AGN at $z \sim 1$ reside, the space density is $\Phi_{\mathrm{H}}(z)=10^{-3} h^{3} \mathrm{Mpc}^{-3}$ (see Fig. 9). For the cosmology adopted here the Hubble time at $z=1$ is $\sim 6.3 \mathrm{Gyr}$.

The comoving space density of XMM-COSMOS AGN has been estimated by considering literature X-ray luminosity function of AGN selected in the $2-10 \mathrm{keV}$ band, which should therefore include unobscured, as well as moderately obscured, objects as the objects populating our sample. Once accounting for band effects $^{9}$, the median luminosity of our $z \sim 1$ sample $(\log L=$ 43.7 in the $0.5-10 \mathrm{keV}$ band; see Table 1) translates into a 2-10 keV luminosity of $\log L \sim 43.5$. At these luminosities, $z \sim 1 \mathrm{AGN}$ in the La Franca et al. (2005) XLF have a space density of $\sim 3 \times 10^{-4} h^{3} \mathrm{Mpc}^{-3}$. A similar value for the AGN density is obtained when using the XLF by Ueda et al. (2003). Therefore, by considering an AGN density of $\sim 1.8 \times 10^{-4} h^{3} \mathrm{Mpc}^{-3}$ (obtained by rescaling the La Franca et al. space density by the fraction of objects with $I_{\mathrm{AB}}<23$, as is the case for our selection), a duty cycle $t_{Q} / t_{\mathrm{H}}$ of 0.18 is obtained, which translates into an AGN lifetime of $\sim 1.1 \mathrm{Gyr}$.

This estimated lifetime is more than one order of magnitude longer than that estimated by Porciani et al. (2004) for bright optical QSOs at $z \sim 1$ in the 2QZ survey. The difference in the measured lifetime is essentially due to the difference between the space density of XMM-COSMOS AGN and 2QZ QSOs at $z \sim 1\left(\approx 2 \times 10^{-4}\right.$ vs. $\approx 10^{-5} \mathrm{~h}^{3} \mathrm{Mpc}^{-3}$, see Table 1 in Porciani et al. 2004). Such a difference is, on the other hand, expected given the relatively bright limiting magnitude $\left(m_{\mathrm{B}} \sim 20.8\right)$ of the 2QZ sample that, in addition, does not include obscured AGN. The estimated lifetime for XMM-COSMOS AGN is significantly shorter than the $\sim 8$ Gyr time span between $z=1$ and $z=$ 0 . This, in combination with the estimate that XMM-COSMOS AGN will cluster with $r_{0}=8 h^{-1} \mathrm{Mpc}$ at $z=0$, depicts a consistent scenario in which XMM-COSMOS AGN will switch off by $z=0$, leaving relic (dormant) supermassive black holes in local elliptical galaxies.

\section{Conclusions}

We have studied the clustering properties of 538 moderately luminous AGN at $z=0.2-3$ in the $2 \mathrm{deg}^{2}$ COSMOS field, selected in the X-rays and spectroscopically identified to $I_{\mathrm{AB}}<23$. Our main results can be summarized as follows:

1. The projected correlation function $w\left(r_{\mathrm{p}}\right)$ on scales $r_{\mathrm{p}}=$ 0.3-40 $h^{-1}$ Mpc can be approximated by a power law with correlation length $r_{0}=8.6 \pm 0.5 h^{-1} \mathrm{Mpc}$ and slope $\gamma=1.9 \pm 0.1$ (Poisson errors; bootstrap errors are a factor of $\sim 2$ larger). This represents the most significant measurement of clustering of X-ray selected AGN to date.

2. Part of the signal, in particular an excess on projected scales $r_{\mathrm{p}}=5-15 h^{-1} \mathrm{Mpc}$, is due to a large scale structure at $z=0.36$. When excluding this structure or computing $w\left(r_{\mathrm{p}}\right)$ for objects in a narrower redshift interval around $z \sim 1$, the correlation length decreases to $r_{0}=5-6 h^{-1} \mathrm{Mpc}$, similar to what is observed in large samples of optically selected QSOs at the same redshift.

3. Objects with different absorption properties do not show significant evidence for different clustering properties. Broad line AGN are consistent with inhabiting the same environments of non-broad line AGN. Similar results are obtained when considering X-ray absorbed and X-ray unabsorbed AGN.

$\overline{9}$ an X-ray photon index of $\Gamma=1.9$ is assumed.
4. No significant difference is found in the clustering properties of objects at redshifts below or above 1 .

5. The correlation length measured for XMM-COSMOS AGN at $z \sim 1$ is similar to that of early type galaxies and luminous infrared galaxies at the same redshift. This, in agreement with other studies, suggests that $z \sim 1$ moderately luminous AGN are found preferentially in massive $\left(M_{\star} \gtrsim 3 \times 10^{10} M_{\odot}\right)$ galaxies.

6. By using public halo catalogs from the Millennium simulation, we estimated XMM-COSMOS AGN to reside within dark matter halos of mass $M \gtrsim 2.5 \times 10^{12} h^{-1} M_{\odot}$.

7. According to a simple conserving scenario for clustering evolution, the relics of $z \sim 1$ AGN are expected to be hosted by local bright $L \sim L_{\star}$ ellipticals by $z=0$.

8. By combining the number density of XMM-COSMOS AGN with that of the hosting dark matter halos, we estimated an AGN duty cycle of 0.1 , which translates into an AGN lifetime of $\sim 1$ Gyr. The estimated lifetime is more than one order of magnitude longer than estimated for optically bright QSOs at the same redshift. This is mainly due to the higher number density of AGN found in X-ray selected samples.

Acknowledgements. This work is based on observations obtained with XMM-Newton, an ESA Science Mission with instruments and contributions directly funded by ESA Member States and the USA (NASA). We gratefully acknowledge the contribution of the entire COSMOS collaboration (http:// www.astro.caltech.edu/ cosmos). In Italy, the XMM-COSMOS projects is supported by ASI-INAF and PRIN/MIUR under grants I/023/05/00 and 200602-5203. The zCOSMOS ESO Large Program Number 175.A-0839 is acknowledged. R.G. thanks Carlo Nipoti, Federico Marulli, Enzo Branchini, and Lauro Moscardini for stimulating discussions. The referee is acknowledged for providing useful comments. The Millennium Simulation databases used in this paper and the web application providing online access to them were constructed as part of the activities of the German Astrophysical Virtual Observatory.

\section{References}

Adelberger, K. L., \& Steidel, C. C. 2005, ApJ, 630, 50

Alexander, D. M., Bauer, F. E., Brandt, W. N., et al. 2003, AJ, 126, 539

Alexander, D. M., Smail, I., Bauer, F. E., et al. 2005, Nature, 434, 738

Basilakos, S., Georgakakis, A., Plionis, M., \& Georgantopoulos, I. 2004, ApJ, 607, L79

Brandt, W. N., \& Hasinger, G. 2005, ARA\&A, 43, 827

Brusa, M., Comastri, A., Mignoli, M., et al. 2003, A\&A, 409, 65

Brusa, M., Zamorani, G., Comastri, A., et al. 2007, ApJS, 172, 353 Cappelluti, N., Hasinger, G., Brusa, M., et al. 2007, ApJS, 172, 341 Cappelluti, N., Brusa, M., Hasinger, G., et al. 2009, A\&A, submitted Carrera, F. J., Barcons, X., Fabian, A. C., et al. 1998, MNRAS, 299 Carrera, F. J., Ebrero, J., Mateos, S., et al. 2007, A\&A, 469, 27

Carroll, S. M., Press, W. H., \& Turner, E. L. 1992, ARA\&A, 30, 499 Cavaliere, A., \& Vittorini, V. 2000, ApJ, 543, 599

Chapman, S. C., Blain, A. W., Ivison, R. J., \& Smail, I. R. 2003, Nature, 422, 695

Coil, A. L., Davis, M., Madgwick, D. S., et al. 2004, ApJ, 609, 525

Coil, A. L., Hennawi, J. F., Newman, J. A., et al. 2007, ApJ, 654, 115

Colless, M. M., Dalton, G. B., Maddox, S. J., et al. 2001, MNRAS, 328, 1039

Constantin, A., \& Vogeley, M. S. 2006, ApJ, 650, 727

Croom, S. M., Boyle, B. J., Shanks, T., et al. 2005, MNRAS, 356, 415

Da Angela, J., Outram, P. J., Shanks, T., et al. 2005, MNRAS, 360, 1040

Daddi, E., Alexander, D. M., Dickinson, M., et al. 2007, ApJ, 670, 173

Davis, M., \& Peebles, P. J. E. 1983, ApJ, 267, 465

Elbaz, D., Daddi, E., Le Borgne, D., et al. 2007, A\&A, 468, 33

Elvis, M., Wilkes, B. J., McDowell, J. C., et al. 1994, ApJS, 95, 1

Elvis, M., Civano, F., Vignali, C., et al. 2009, ApJS, submitted

Fabian, A. C. 1999, MNRAS, 308, L39

Ferrarese, L., \& Merritt, D. 2000, ApJ, 539, L9

Ferrarese, L., \& Ford, H. 2005, SSRv, 116, 523

Fisher, K., Davis, M., Strauss, M. A., Yahil, A., \& Huchra, J. 1994, MNRAS, 266, 50

Francke, H., Gawiser, E., Lira, P., et al. 2008, ApJ, 673, L13

Fry, J. N. 1996, ApJ, 461, L65

Gandhi, P., Garcet, O., Disseau, L., et al. 2006, A\&A, 457, 393

Gavignaud, I., Wisotzki, L., Bongiomo, A., et al. 2008, A\&A, 492, 637 
Gebhardt, K., Bender, R., Bower, G., et al. 2000, ApJ, 539, L13

Gehrels, N. 1986, ApJ, 303, 336

Georgakakis, A., Nandra, K., Laird, E. S., et al. 2007, ApJ, 660, L15

Gilli, R., Daddi, E., Zamorani, G., et al. 2005, A\&A, 430, 811

Gilli, R., Comastri, A., \& Hasinger, G. 2007a, A\&A, 463, 79

Gilli, R., Daddi, E., Chary, R., et al. 2007b, A\&A, 475, 83

Gioia, I., Henry, J. P., Mullis, C. R., et al. 2003, ApJS, 149, 29

Granato, G., De Zotti, G., Silva, L., Bressan, A., \& Danese, L. 2004, ApJ, 600, 580

Grazian, A., Negrello, M., Moscardini, L., et al. 2004, AJ, 127, 592

Hasinger, G. 2008, A\&A, 490, 905

Hasinger, G., Miyaji, T., \& Schmidt, M. 2005, A\&A, 441, 417

Hasinger, G., Cappelluti, N., Brunner, H., et al. 2007, ApJS, 172, 29

Hopkins, P. F., Hernquist, L., Cox, T. J., et al. 2006, ApJS, 163, 1

Kampczyk, J. S., Lilly, S. J., Carollo, C. M., et al. 2007, ApJS, 172, 329

Kartaltepe, J. S., Sanders, D. B., Scoville, N. Z., et al. 2007, ApJS, 172, 320

Kauffmann, G., \& Haehnelt, M. G. 2000, MNRAS, 311, 576

Kauffmann, G., \& Haehnelt, M. G. 2002, MNRAS, 332, 529

Kauffmann, G., Colberg, J. M., Diaferio, A., \& White, S. D. M. 1999, MNRAS, 307,529

Kauffmann, G., White, S. D. M., Heckman, T. M., et al. 2004, MNRAS, 353, 713

Kerscher, M., Szapudy, I., \& Szalay, A. 2000, ApJ, 535, L13

Kitzbichler, M., \& White, S. 2006, MNRAS, 2007, 376, 2

La Franca, F., Andreani, P., \& Cristiani, S. 1998, ApJ, 497, 529

La Franca, F., Fiore, F., Comastri, A., et al. 2005, ApJ, 635, 864

Landy, S. D., \& Szalay, A. S. 1993, ApJ, 412, 64

Lehmer, B. D., Brandt, W. N., Alexander, D. M., et al. 2005, ApJS, 161, 21

Le Fevre, O., Mellier, Y., McCracken, H. J., et al. 2004, A\&A, 417, 839

Li, C., Kauffmann, G., Wang, L., et al. 2006, MNRAS, 373, 457

Li, C., Kauffmann, G., Heckman, T. M., White, S. D. M., \& Jing, Y. P. 2008, MNRAS, 385, 1915

Lidz, A., Hopkins, P. F., Cox, T. J., Hernquist, L., \& Robertson, B. 2006, ApJ, 641,41

Lilly, S. J., Le Fèvre, O., Renzini, A., et al. 2007, ApJS, 172, 70

Mainieri, V., Rosati, P., Tozzi, P., et al. 2005, A\&A, 437, 805

Mainieri, V., Hasinger, G., Cappelluti, N., et al. 2007, ApJS, 172, 368

Malkan, M. A., Gorjian, V., \& Tam, R. 1998, ApJS, 117, 25

Marconi, A., Risaliti, G., Gilli, R., et al. 2004, MNRAS, 351, 169

Martini, P., \& Weinberg, D. H. 2001, ApJ, 547, 12

Marulli, F., et al. 2009, MNRAS, submitted

Marzke, R. O., Geller, M. J., Da Costa, L. N., \& Huchra, J. P. 1995, AJ, 110, 477

McLure, R. J., \& Dunlop, J. S. 2004, MNRAS, 352, 1390

Meneux, B., Le Fèvre, O., Guzzo, G., et al. 2006, A\&A, 452, 387

Miyaji, T., Zamorani, G., Cappelluti, N., et al. 2007, ApJS, 172, 396

Mo, H. J., Jing, Y. P., \& Börner, G. 1992, ApJ, 392, 452

Moscardini, L., Coles, P., Lucchin, F., \& Matarrese, S. 1998, MNRAS, 299, 95

Mullis, C. R., Henry, J. P., Gioia, I. M., et al. 2004, ApJ, 617, 192

Murray, S., Kenter, A., Forman, W. R., et al. 2005, ApJS, 161, 1

Nandra, K., Laird, E. S., Adelberger, K., et al. 2005, MNRAS, 356, 568

Noeske, K. G., Weiner, B. J., Faber, S. M., et al. 2007, ApJ, 660, L43

Nusser, A., \& Davis, M. 1994, ApJ, 421, L1

Osmer, P. S. 1981, ApJ, 247, 762

Padmanabhan, N., et al. 2008, MNRAS, submitted [arXiv:0802 .2105]

Peebles, P. J. E. 1980, The Large Scale Structure of the Universe (Princeton: Princeton Univ. Press)

Pierre, M., Chiappetti, L., Pacaud, F., et al. 2007, MNRAS, 382, 279

Plionis, M., Rovilos, M., Basilakos, S., Georgantopoulos, I., \& Bauer, F. 2008, ApJ, 674, L5

Porciani, C., Magliocchetti, M., \& Norberg, P. 2004, MNRAS, 355, 1010

Porciani, C., \& Norberg, P. 2006, MNRAS, 371, 1824

Puccetti, S., Fiore, F., D'Elia, V., et al. 2006, A\&A, 457, 501

Rosati, P., Giacconi, R., Gilli, R., et al. 2002, ApJ, 566, 667

Salvato, M., Hasinger, G., Ilbert, O., et al. 2009, ApJ, 690, 1250

Sanders, D. B., \& Mirabel, I. F. 1996, ARA\&A, 34, 749

Sanders, D. B., Salvato, M., Aussel, H., et al. 2007, ApJS, 172, 86

Schinnerer, E., Smolč ić, V., Carilli, C. L., et al. 2007, ApJS, 172, 46

Scoville, N., Aussel, H., Brusa, M., et al. 2007a, ApJS, 172, 1

Scoville, N., Abraham, R. G., Aussel, H., et al. 2007b, ApJS, 172, 38

Shanks, T., Fong, R., Boyle, B. J., \& Peterson, B. A. 1987, MNRAS, 277, 739
Shankar, F., Salucci, P., Granato, G. L., De Zotti, G., \& Danese, L. 2004, MNRAS, 354, 1020

Shen, Y., Strauss, M. A., Oguri, M., et al. 2007, AJ, 133, 2222

Silverman, J. D., Green, P. J., Barkhouse, W. A., et al. 2008a, ApJ, 679, 118

Silverman, J. D., Lamareille, F., Maier, C., et al. 2008b, ApJ, in press [arXiv: 0810.3653]

Spergel, D. N., Bean, R., Doré, O., et al. 2007, ApJS, 170, 377

Springel, V., White, S. D. M., Jenkins, A., et al. 2005, Nature, 435, 629

Tacconi, L. J., Genzel, R., Smail, I., et al. 2008, ApJ, 680, 246

Taniguchi, Y., Scoville, N., Murayama, T., et al. 2007, ApJS, 172, 9

Tozzi, P., Gilli, R., Mainieri, V., et al. 2006, A\&A, 451, 457

Trump, J. R., Impey, C. D., McCarthy, P. J., et al. 2007, ApJS, 172, 383

Ueda, Y., Akiyama, M., Ohta, K., \& Miyaji, T. 2003, ApJ, 598, 886

Ueda, Y., Watson, M. G., Stewart, I. M., et al. 2008, ApJS, 179, 124

Vikhlinin, A., \& Forman, W. 1995, ApJ, 455, L109

Yang, Y., Mushotzky, R. F., Barger, A. J., \& Cowie, L. L. 2006, ApJ, 645, 68

York, D. C., Adelman, J., Anderson, J. E., Jr., et al. 2000, AJ, 120, 1579

Yu, Q., \& Tremaine, S. 2002, MNRAS, 335, 965

Zamojski, M. A., Schiminovich, D., Rich, R. M., et al. 2007, ApJS, 172, 468

Zehavi, I., Blanton, M. R., Frieman, J. A., et al. 2002, ApJ, 571, 172

Zehavi, I., Weinberg, D. H., Zheng, Z., et al. 2004, ApJ, 608, 16

1 INAF - Osservatorio Astronomico di Bologna, Via Ranzani 1, 40127 Bologna, Italy

e-mail: roberto.gilli@oabo.inaf.it

2 Instituto de Astronomía, Universidad Nacional Autónoma de México, Ensenada, México (mailing address: PO Box 439027, San Ysidro, CA, 92143-9027, USA)

3 Institute of Astronomy, Swiss Federal Institute of Technology (ETH Hönggerberg), 8093, Zürich, Switzerland

4 Max-Planck-Institut für extraterrestrische Physik, Postfach 1312, 85741 Garching, Germany

5 European Southern Observatory, Karl-Schwarzschild-Strasse 2, Garching 85748, Germany

6 Laboratoire AIM, CEA/DSM - CNRS - Université Paris Diderot, DAPNIA/SAp, Orme des Merisiers, 91191 Gif-sur-Yvette, France

7 Harvard-Smithsonian Center for Astrophysics, 60 Garden Street, Cambridge, MA 02138, USA

8 INAF - Osservatorio Astronomico di Roma, via di Frascati 33, 00040 Monte Porzio Catone (Roma), Italy

9 California Institute of Technology, MC 105-24, 1200 East California Boulevard, Pasadena, CA 91125, USA

10 Dipartimento di Astronomia, Università degli Studi di Bologna, Via Ranzani 1, 40127 Bologna, Italy

11 Steward Observatory, University of Arizona, Tucson, AZ 85721, USA

12 Institut d'Astrophysique de Paris, 98 bis Boulevard Arago, 75014 Paris, France

13 Research Center for Space and Cosmic Evolution, Ehime University, Bunkyo-cho 2-5, Matsuyama 790-8577, Japan

14 Laboratoire d'Astrophysique de Toulouse-Tarbes, Université de Toulouse, CNRS, 14 avenue E. Belin, 31400 Toulouse, France

15 Laboratoire d'Astrophysique de Marseille, CNRS-Université de Provence, Traverse du Siphon, BP 8, 13012 Marseille, France

16 Dipartimento di Astronomia, Università di Padova, Vicolo Osservatorio 2, 35122 Padova, Italy

17 INAF - Istituto di Astrofisica Spaziale e Fisica Cosmica, Via Bassini 15, 20133 Milan, Italy

18 INAF - Osservatorio Astronomico di Brera, 23807 Merate (LC), Italy

19 University of California, Lawrence Berkeley National Laboratory, 1 Cyclotron Rd, Berkeley, CA 94720, USA

20 Centre de Physique Théorique, UMR 6207 CNRS-Université de Provence, Case 907, 13288 Marseille, France 